Florida International University FIU Digital Commons

9-7-2016

\title{
Memory, Processing Speed, and the Effects of Cognitive Exercise on the Aging Brain
}

\author{
Alexis D. Yero \\ Communication and Science Disorders at Florida International Univeristy, ayero001@fiu.edu
}

DOI: $10.25148 /$ etd.FIDC001172

Follow this and additional works at: https://digitalcommons.fiu.edu/etd

Part of the Speech Pathology and Audiology Commons

\section{Recommended Citation}

Yero, Alexis D., "Memory, Processing Speed, and the Effects of Cognitive Exercise on the Aging Brain" (2016). FIU Electronic Theses and Dissertations. 3062.

https://digitalcommons.fiu.edu/etd/3062

This work is brought to you for free and open access by the University Graduate School at FIU Digital Commons. It has been accepted for inclusion in FIU Electronic Theses and Dissertations by an authorized administrator of FIU Digital Commons. For more information, please contact dcc@fiu.edu. 


\section{FLORIDA INTERNATIONAL UNIVERSITY}

Miami, Florida

\section{MEMORY, PROCESSING SPEED, AND THE EFFECTS OF COGNITIVE EXERCISE ON THE AGING BRAIN}

A thesis submitted in partial fulfillment of the

requirements for the degree of

MASTER OF SCIENCE

in

SPEECH LANGUAGE PATHOLOGY

by

Alexis Yero

2016 


\section{To: Dean Ora Strickland}

Nicole Wertheim College of Nursing \& Health Sciences

This thesis, written by Alexis Yero, and entitled Memory, Processing Speed, and the Effects of Cognitive Exercise on the Aging Brain, having been approved in respect to style and intellectual content, is referred to you for judgment.

We have read this thesis and recommend that it be approved.

Alfredo Ardilla

Eliane Ramos

Monica Hough, Major Professor

Date of Defense: September 7, 2016

The thesis of Alexis Yero is approved.

Dean Ora Strickland

Nicole Wertheim College of Nursing \& Health Sciences

Andrés G. Gil

Vice President for Research and Economic Development and Dean of the University Graduate School

Florida International University, 2016 
(C) Copyright 2016 by Alexis Yero

All rights reserved. 


\section{DEDICATION}

This thesis is warmly dedicated

to my grandmother, Victoria.

She was an immensely strong, and courageous, woman;

one who not only taught me how to work hard,

but how to play even harder.

I love and miss you, Yiayia. 


\section{ACKNOWLEDGMENTS}

I would like to thank our Heavenly Father who has provided me with the strength, determination, and grace to conduct this study. I would like to thank my beautiful mother, my loudest supporter; without her positivity, and unconditional motherly love, this study would not have been possible. I would like to thank Dr. Fabian Redler, for inspiring my passion for cognitive skills therapies. Lastly, I would like to thank my wonderful Thesis Chair, Dr. Monica Hough, for her firm but gentle guidance, dedication, and her continuous support. 


\section{ABSTRACT OF THE THESIS \\ MEMORY, PROCESSING SPEED, AND THE EFFECTS OF COGNITIVE EXERCISE \\ ON THE AGING BRAIN}

by

Alexis Yero

Florida International University, 2016

Miami, Florida

\section{Professor Monica Hough, Major Professor}

The purpose of the current study was to investigate, and expain, the effects of an intervention known as "The Five Task Approach" (TFTA); a cognitive intervention hereby utilized within the realm of the geriatric population, as a means of taxing and strengthening cortical areas associated with memory, and visual processing. This study revealed that even short-term exposure to cognitive activities, and therapeutic cueing known to tax areas connected to visual perception, may have an effect on one's global cognition, generalized memory, and the accuracy of one's visual perception. It was demonstrated that even brief cognitive intervention geared at taxing cortical areas associated with memory and visual processing, in conjunction with the therapuetic cueing utilized in this study, has the potential to significantly increase participant performance in terms of global cognitive function, including skills associated with executive functioning, working memory, visual processing, visual processing speed, auditory processing, and global cognitive status. 


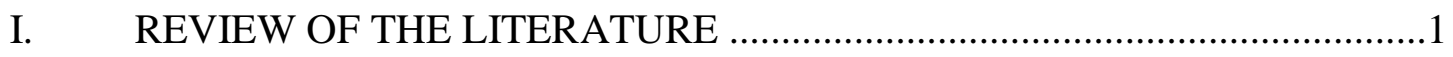

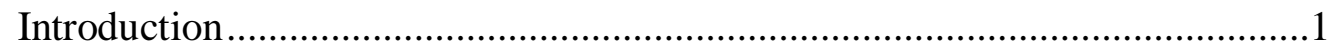

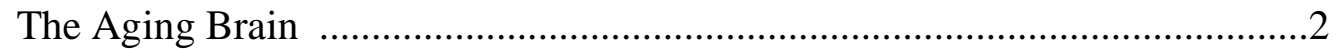

Attention and Memory in Aging ....................................................................

Aging and Cognitive Processing Speed ......................................................

Brain Plasticity and Response to Cognitive Exercise ........................................9

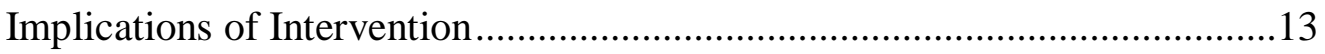

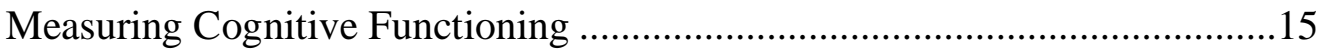

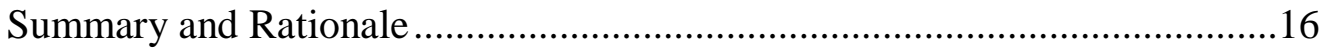

Plan of Study and Experimental Questions ...................................................18

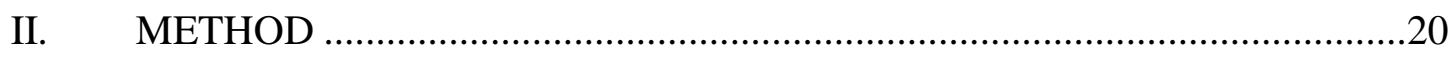

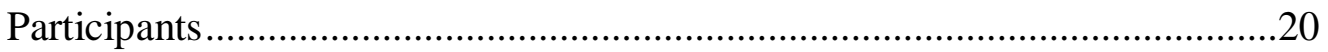

Pre-Experimental Testing ......................................................................22

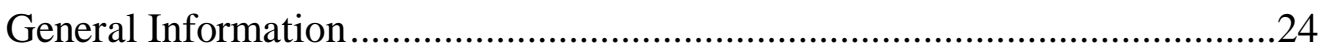

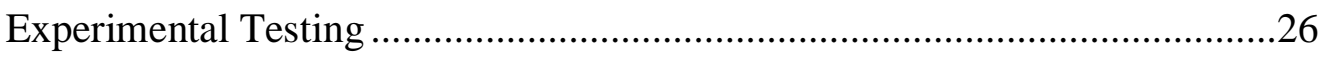

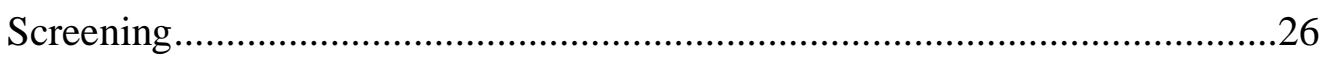

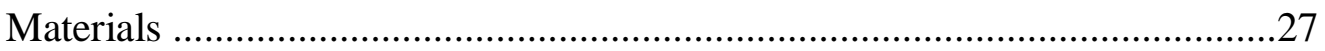

Experimental Procedures/Protocols …………………................................28

Interventional Cueing ...................................................................................

Design and Data Analysis ..............................................................................

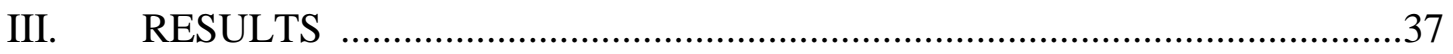

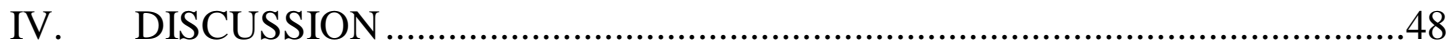

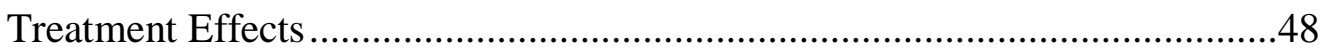

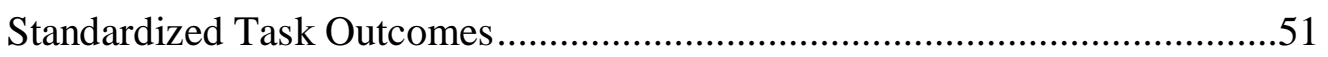

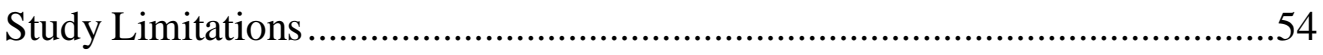

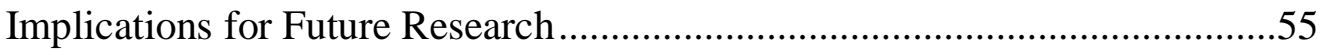

Summary and Conclusions ............................................................................56 
REFERENCES

APPENDICES 


\section{LIST OF TABLES}

TABLE

PAGE

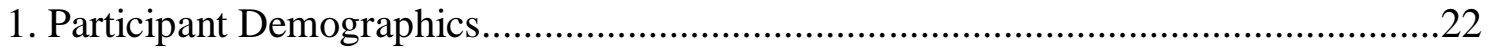

2. Baseline and Post-Treatment Data for the 5 Experimental Tasks.....................39

3. Dementia Rating Scale-2 Pre/Post Scores.........................................44

4. Motor Free Visual Perception Test-4 Pre/Post Scores..............................46

5. Mini Mental State Examination Pre/Post Scores.......................................................47 


\section{LIST OF FIGURES}

FIGURES

PAGE

1. Active Participant Performance on The Number Pair Activity Task.........................40

2. Active Participant Performance on The Flash Card Memory Drill Task.............40

3. Active Participant Performance on The Sequential Number Memory (Reverse Order) Task...................................................41

4. Active Participant Performance on The Letter Circle Task.....................................41

5. Active Participant Performance on The Detailed Picture Question Task..............42 


\section{CHAPTER 1}

\section{REVIEW OF THE LITERATURE}

\section{Introduction}

As individuals grow older, it is commonplace to hear grievances about decreased mental performance in terms of speed and cognition. Cognitive changes and aging are often considered to be inevitable outcome that all humans must endure throughout an adult lifespan. It has been assumed that declining cognitive skills are to be expected with normal aging, and that it is unavoidable as one makes the transition in to the geriatric realm of life (Eramudugolla, Cherbuin, Easteal, Jorm, \& Anstey, 2013; Williams, 2011). However, is cognitive skill degeneration always an inevitable and irreversible process? With a growing geriatric population throughout the world, it is vital that researchers make a diligent effort to identify modern therapeutic techniques and interventions that may potentially prolong and improve the effectiveness of cognitive functioning in one's elder years (Leff, Kao, \& Ritchie, 2015).

A small, but growing body of research indicates that stimulating certain cortical area via cognitive function tasks geared at specific functions can help prolong and improve some of the signs associated with the aging brain (Nouchi et al., 2012; Williams, 2011). The present study aims to investigate the effects of performing cognitive tasks that engage working memory abilities, visual processing abilities and the subsequent improvement on cognitive batteries measuring visual processing speed and visual and auditory memory. Initially, information on the aging brain, and the cognitive processes affected, will be addressed. These processes involve neurological skills that aide in attention, memory, processing speed, and influences on neuronal plasticity. This 
discussion will be followed by presentation of information on implications for intervention and measuring cognitive functions. The review of the literature will conclude with the summary, rationale, plan of study, and experimental questions for the current investigation.

\section{The Aging Brain}

It is understood that cognitive decline can lead to the loss of one's independent living, and daily functioning in elder years (Glisky, 2007, Zelinski \& Gilewski, 2004). In addition to overt negative consequences, self-reported cognitive declines are also associated with the persistence of covert depression and anxiety in older individuals. It has been noted that this population lives with a fear of forgetfulness, and often feels powerless against the cognitive aging process (Butler \& Lewis, 1986; Zelinski \& Gilewski, 2004). Decline may lead to difficulty with medication management, meal preparation and planning, and financial planning (Brown, Brockmole, Gow, \& Deary, 2012; Williams, 2011).

Older individuals may be reluctant to report symptoms associated with cognitive decline for fear that confession may result in being stuck with a confusing "label", or that they may be treated as if they are no longer cognitively intact (Anderson, Murphy, \& Troyer, 2012; Williams, 2011). If and when these symptoms are reported, many medical professionals may simply attribute the negative signs to "old age", and discharge the patient with recommendations to do aerobic exercises, and to eat a balanced diet (Anderson et al., 2012). While incorporating healthy eating habits and daily exercise in to one's lifestyle are invaluable recommendations (Anderson et al., 2012); too often, health professionals may neglect to advocate for the patient to include exercises of the brain in 
his or her daily routine. As research continues to reveal the benefits of exercising one's cognitive functioning skills, more clinical instruments are being developed to incorporate novel ways of sustaining as well as enhancing cognitive proficiency (Brown et al., 2012). Thus, more evidence-based research is needed to support the efficacy of such recommendations.

In older adulthood, one's processing speed is contemporaneously correlated with the strength of his or her overall cognitive abilities (Deary, Whalley, Lemmon, Crawford, $\&$ Starr, 2000). In fact, Brown and colleges (2012) found that an individual's visual working memory is related to overall processing speed, as well as spatial working memory. One of the most common physical cerebral changes connected to aging is atrophy of the brain, which is amplified by shrinking of certain cortical areas in the brain (Anderson et al., 2012). Atrophy is often associated with a decline in cognitive functioning, especially with skills mediated by the prefrontal cortex (Anderson et al., 2012). Despite the evidence based treatments known to defer the atrophy process (Roizen $\& \mathrm{Oz}, 2007)$, the research often goes over looked, and underutilized. Fotuhi, Do, and Jack (2012) reported that there is now a positive correlation between performing cognitive brain tasks and a growth of the hippocampus, a structure of the brain that has shown to be strongly tied to human memory. As one of the first studies to investigate the growth of cortical structures, it is now understood that just as cortical and subcortical structures can shrink, they can also be enhanced (Fotuhi, et al., 2012).

As the human brain ages the most prevalent degree of atrophy is seen in the frontal and temporal lobes. Significant atrophic changes may also be observed in the medial parietal precuneus area, retrosplenial and posterior cingulate cortices (Fjell, 
McEvoy, Holland, Dale, \& Walhovd, 2014). Many of the areas of the human brain associated with writing, speech, reasoning, sequencing, comprehension, visual memory, working memory, long term memory, as well as other cognitive tasks necessary to maintain independent functioning, are tasks that are mediated by structures situated within these respective lobes (Fjell et al., 2014).

\section{Attention and Memory in Aging}

Perhaps the two most prevailing cognitive functions associated with cognitive decline in the elderly are overall attention, in terms of processing deterioration of both visual and auditory information, as well as general working memory (Glisky, 2007). That is, as individuals age, their ability to focus, and to retain adequate visual and auditory short-term information is compromised (Brown et al., 2012; Gilsky, 2007; Karbach, 2014). Working memory can be understood as skills that enable an individual to manipulate and retain stimulus information simultaneously (Brown et al., 2012). While these skills may deteriorate with age, that does not necessarily mean that once an individual experiences declines that they are destined to continue to show signs of cerebral degeneration indefinitely (Roizen \& Oz, 2007). A study conducted by Van Muijden, Band, and Hommel (2012) involved of the administration of 5 different brain games targeting the areas of working memory, set shifting, response inhibition, attention, and inductive reasoning in individuals between the ages of 60 and 77 . Results showed that engaging in these cognitive brain games substantially improved working memory performance of the adults in the active participant condition. Participants who engaged in the videogame interventions, integrating five different games, scored significantly higher 
on post measures when compared to those improvements gained by the group assigned to simply answer computerized questions after viewing an informative video.

It also was found that the games had significant generalized effects to both fluid intelligence, inductive reasoning, and inhibition, when compared to the control group as indicated by post measures on the top-Signal task and the Raven-Standard Progressive Matrices (Van Muijden et al., 2012). Bissig and Lustig (2007) found that in older individuals, the degree of one's self-initiation and that active participation in taxing one's practice of self-initiation on intentional encoding tasks, was predictive of improved performance with the recollection memory-training task.

Sufficient and functional attention and memory skills are crucial factors one should consider when deciding if an individual has the capacity to care for his or her self, and to safely live independently. As of 2011, assisted living homes were the fastest growing residential option for seniors, with a projection of a two-fold growth by 2021 (Williams, 2011). A concern with increasing memory lapses, and forgetfulness, were among the top responses when residents were questioned (Williams, 2011). Assisted living homes provide older adults with a more autonomous style of living than the traditional nursing home, and enable them to remain independent for as long as reasonably possible. As one's memory is pivotal to self-care, it is prudent that such facilities make continued cognitive exercise a key component in the individual's life. According to meta-analysis data compiled by Sitzer, Twamley, \& Jeste (2006), assisted living homes that promoted cognitive skills such as a resident's learning abilities, attention, executive functions, general problem solving, and memory had fewer cases of resident's reporting/exhibiting cognitive decline. This is indicative of the fact that 
promoting cognitive skills into one's elderly years is beneficial for continued neuronal health, as well as prevention of memory decline. Sitzer et al.'s (2006) meta-analysis is suggestive that assisted living patients with or without mild cognitive decline may benefit from cognitive intervention. Engaging in tasks that recruit use of cortical areas associated with memory may enable individuals to maintain current level of functioning, and support cognitive skills that are adequate for activities of daily living.

\section{Aging and Cognitive Processing Speed}

In addition to the declining memory and attention skills, cognitive processing speed is another frequently reported complaint among older adults. Specifically, individuals report that it takes them longer for information to process, in that they may require more time to receptively understand visual and auditory information that is presented to them (Glisky, 2007). The slowing of cognitive processing speed and reduced reaction time also has been related to depression in this population (Butler \& Lewis, 1986). One can understand why reduced processing speed would have negative implications on one's quality of life. By activating, and thereby strengthening areas of the brain that are associated with the specific tasks, one can significantly improve his or her daily lifestyle and functional skills (Roizen \& Oz, 2007). As previously mentioned, atrophic changes of the medial parietal precuneus area, retrosplenial and posterior cingulate cortices may lead to deterioration of processing skills, as well as one's memory skills (Fjell, et al., 2014). In other words, if one does not exercise cortical areas by engaging in activities that activate the brain, cognitive decline is more likely to be observed. Research has shown that cognitive processing speed is one of the more 
susceptible cognitive dexterities known to significantly react to brain exercise intervention (Edwards et al., 2005). Albeit, a growing body of research has indicated that in many instances, cognitive deterioration need not be irreversible; rather, individuals can practice daily cognitive exercises as a means of keeping cortical functioning (Bissig and Lustig, 2007; De Gobbi Porto, Fox, Tusch, Sorond, Mohammed, and Daffner, 2015; Edwards, Wadley, Vance, Wood, Roenker, and Ball, 2005; Fotuhi, Do, and Jack, 2012; Nouchi et al., 2012, Osaka, Yaoi, Osaka, Katsuhara, and Osaka, 2012; Rebok, et al., 2014; Roizen \& Oz, 2007; Sitzer, Twamley, \& Jeste, 2006; Smith, Housen, Yaffe, Ruff, Kennison, Mahncke, and Zelinski, 2009; Van Muijden, Band, and Hommel, 2012; Williams, 2011).

A randomized control study conducted by Nouchi et al. (2012) concluded that cognitive exercise has efficacious implications for the geriatric population. In the aforementioned study, 36 participants were recruited to study the effects of playing two cognitive training games, "Brain Age", or "Tetris" for approximately 200 minutes over the course of 4 weeks. When compared to the control condition, the active participants in both the "Brain Age" and "Tetris" groups performed significantly higher on postintervention measures of executive functioning as measured by the Frontal Assessment Battery and the Trail Making Test- B, as well as demonstrated improvements in processing speed, as measured by Digit Symbol Coding and Symbol Search tasks (Nouchi et al., 2012). That is, active participants showed increases in cognitive processing speeds, as well as executive functioning skills (Nouchi et al., 2012). The results are also indicative that brain game exercises, even when done for short-term periods of time, may yield results that enhance neurological functioning in a number of 
untrained aspects. Nouchi et al. (2012) found that processing speed was one of the skills shown to improve significantly. Processing speed is an essential cognitive skill affecting the effectiveness of one's working memory skills (Glisky, 2007). Thus, the importance of these findings is understandably relative to aiding clinicians with design and implementation of intervention, when working with older clients warranting cognitive rehabilitation.

The body of evidence involved with reviewing the efficacy of various types of brain exercises is increasing. More institutions are taking initiative to educate the geriatric population about the importance of maintaining cognitively agility by purposefully engaging in tasks that enhance cognitive activity. It is common for the geriatric population to be encouraged to do tasks such as cross word puzzles, search-awords, and Sudoku puzzles to activate cortical areas involved with the skills needed to perform the tasks. Websites such as the American Association of Retired Persons (AARP) now encourage, and provide online resources and materials for seniors to play diversified brain games at their leisure (Brain Games, 2015). Currently, the AARP website offers user friendly, online games that target processing speed, memory, attention, and language skills.

\section{Brain Plasticity and Response to Cognitive Exercise}

Even in older adults, the brain retains plasticity, and proves to stand malleable, even with the passage of time (Edwards et al., 2005). This indicates that the brain should therefore be susceptible to strengthening, and growth, when exercised (Edwards et al., 2005). Cognitive neuroplasticity is a vital function that permits the human brain to modify, alter, and restructure cortical structures as a response to environmental taxing in 
the form of strengthening, weakening, eliminating, or creating new synaptic connections, thereby allowing for neurogenesis (De Gobbi Porto et al., 2015). De Gobbi Porto and colleagues (2015) conducted one of the first in vivo imaging of older adults, all over of the age of 65, via an electroencephalogram (EEG). EEG probes were placed on and around the participant's orbital sockets, as well as on the upper and lower mastoids. Simultaneously, visual evoked potential signals (VEPs) were presented. The researchers concluded that after only 2 minutes of exposure to the VEPs, there were notable neuroplastic changes in the participants, including changes in the efficacy of synaptic connections, when compared with post exposure images (De Gobbi Porto, 2015).

Research conducted by Osaka et al. (2012) demonstrated positive cortical effects associated with practicing brain activities with healthy elderly adults. In the study, participants were administered the Stroop Task, which targets visual processing skills, reaction time, and working memory. The results of fMRIs following the task revealed that participants had significant increases in the activation of the anterior cingulate cortex, the left inferior parietal lobule, the left dorsolateral prefrontal cortex, and the precuneus regions. On the other hand, the control group, who had not participated with the task, showed no remarkable increases in these cortical areas (Osaka et al., 2012).

Furthermore, a study conducted by Smith et al. (2009) demonstrated that generalization effects were generalized to untrained standardized measures, particularly in the realms of memory and attention. Specifically, Smith et al. (2009) recruited 487 adults over the age of 65, who were diagnosed with cognitive impairments. During intervention, participants utilized brain plasticity-based computerized cognitive training programs for 40 hours, over the course of 8 consecutive weeks. The six computer 
exercises were designed to improve speed and accuracy of the processing of auditory information. It was hypothesized that generalized effects would be seen in realms of cognitive functioning, not specifically targeted by the six computer games (Smith et al., 2009). Researchers concluded that there were in fact generalized cognitive effects in the domains of memory and attention, as well as participants self-reported statements of overall improved cognitive functioning. In addition, participants involved in the cognitive training intervention performed significantly better on measures directly related to the trained tasks (Smith et al., 2009).

Studies such as the aforementioned are supportive of the notion that engaging in cognitive brain games in one's elderly years can increase the activity within, and perhaps functioning of, major cortical areas associated with skills needed to stay "sharp and alert." Additional research has found that cognitive skills training has revealed increased activity in the prefrontal, and left lateral temporal regions, which are associated with selfinitiated functional strategies in the elderly (Kirchhoff, Anderson, Smith, Barch, and Jacoby, 2012).

Moreover, federally funded cognitive training research including over 2,800 geriatric individuals with a median age of 74 years was conducted utilizing the “Advanced Cognitive Training for Independent and Vital Elderly" (ACTIVE) intervention to measure the effect size of cognitive training on participant's evaluations on the Instrumental Activities of Daily Living (Rebok, et al., 2014). Similar to other cognitive enhancing interventions, the ACTIVE program was designed to target functions such as processing speed, visual and auditory processing, memory, reasoning, and planning (Rebok et al., 2014). The results of this study have revealed astounding 
findings, indicating that cognitive training intervention had positive carry over effects (in areas of processing speed and reasoning) for 10 years after initial interaction with the intervention (Rebok, et al., 2014). A study conducted by Gaitán et al. (2007) involved participants completing a 12-month intervention utilizing computer-based cognitive therapy $(\mathrm{CBCT})$. The intervention was geared at improving cognitive processes associated with language, memory, attention and concentration, calculations, as well as examining participants' anxiety levels in response to their changing cognitive skills resulting from the aging process. Although participants did not show increases relative to cognitive process proficiency at the end of the study, participants presented with fewer symptoms of anxiety when compared to anxiety measures taken prior to the inclusion of the CBCT, as well as lower levels when contrasted with those reported by the control group (Gaitán et al., 2013).

\section{Implications of Intervention}

Although the results of various cognitive intervention techniques have divergent implications in reference to generalization of specific treatment, nearly all studies allude to the fact that the brain retains plasticity in older adults (Edwards et al., 2005). The empirical results derived from research studies such as the aforementioned, indicate that active cognitive skills training in the elderly population can in fact improve cognitive skills, and quality of effective, self-reliant daily functioning (Smith et al., 2009). The notion that there is an apparent correlation between activating cognitive areas, and improved cognitive skills functioning, promotes the idea that brain plasticity remains responsive with the passage of time (Edwards et al., 2005). Consequently, a decline in 
cognitive functioning does not need to be a continuous, inevitable, or even an irreversible process (Edwards et al., 2005; Smith et al., 2009).

Research supported by Gates and Valenzuela (2010), suggests that cognitive training exercises serve as primary preventive care in reference to prolonging cortical degeneration, and further posit that the increased cognitive activation can slow the progression of degeneration in individual's labeled at risk for dementia. The fact that cognitive training aimed at stimulating neuroplastic pathways (in the already aged brain) can result in generalized improvement effects to other cognitive skill areas that were not targeted (Gates \& Valenzuela, 2010), suggests that brain plasticity is very much present and receptive to intervention, even in one's elder years. Furthermore, Anderson and Grossberg (2014) postulate that one of the most effective ways that an individual can play a role in slowing cognitive decline, and to possibly delay or decrease one's risk of developing Alzheimer's disease, is to continue actively exercising the brain throughout one's life.

Comprehensively, research investigating the efficacy of cognitive brain exercises is suggestive of the fact that neuronal plasticity is viable, and susceptible to interventions aimed at improving various cognitive functions, even in elderly individuals (Edwards et al., 2005; Smith et al., 2009). These interventions may have long-term, generalized improvement effects to higher levels of cognitive functioning (Rebok et al., 2014). Research has shown that brain exercises are correlated with improved scores on selfreported basic functioning measures, as well as improved feelings on one's quality of life (Edwards et al., 2005). However, the scarcity of replicable research in this area (Anderson \& Grossberg, 2014; Glisky, 2007), in reference to the exact treatment, 
protocols, specific characteristics of the interventions, and carry over effects, has prevented the available data from potentially formulating any exhaustive or definitive conclusions.

Despite the fact that the effects of intervention may be short-lived for some participants, the strategies learned, the cues/treatments provided, and the recommendations that the participant receives during the facilitation of engaging in a cognitive brain game, can be utilized post-intervention. These "trained" individuals may rely on these learned strategies and recommendations to better deal with consequences of cognitive decline in the future. Definitive neurocognitive therapeutic research interventions will have enormous implications for the geriatric population as a whole, as modern society continues to promote autonomous functioning for as long as possible.

\section{Measuring Cognitive Functioning}

Neuropsychological batteries are intrinsically performance-based in nature (Harvey, 2012). Performance is typically measured via composite scores, based on the effectiveness of multiple cognitive abilities. These batteries, known to possess high reliability and validity ratings, typically evaluate cognitive skills related to memory, attention, processing speed, reasoning, judgment, problem solving, spatial relations, and language function (Harvey, 2012). An individual's composite scores provide an overall index of the effectiveness of his or her cognitive functioning at a specific point in time. Performance-based, criterion and standardized measures, including the Mini Mental State Examination (MMSE) (Folstein, Folstein, \& McHugh, 1975). the Motor-Free Visual Perception Test-4 (MVPT-4) (Colarusso \& Hammill, 2015), and the Dementia Rating Scale-2 (DRS-2) (Jurica, Leitten, \& Mattis, 2001), are instruments used to evaluate and 
monitor cognitive processes. These measures are established, and known to be valid and reliable (Colarusso \& Hammill, 2015; Folstein et al., 1975; Teng \& Chui, 1987; Jurica et al., 2001). The MMSE is designed to assess one's orientation, immediate recall, attention, delayed verbal recall, naming, repetition, reading, writing, and copying (Folstein et al., 1975). The MVPT-4 assesses one's spatial relationships, visual closure, visual discrimination, visual memory, figure ground, and visual closure (Colarusso \& Hammill, 2015). The DRS-2 is a standardized more complete measure of cognitive functions, including attention, initiation/perseveration, construction, conceptualization, and memory. As additional research confirms that need to address cognitive aging in the elderly population, batteries that address these cognitive functions, specifically working memory tasks, have become more common place as a crucial component in the investigation of potential cognitive changes in the mental capacities of elderly adults (Brown et al., 2012).

\section{Summary and Rationale}

Recent evidence has indicated that engaging in cognitive exercises enhances cognitive skills, such as increasing one's working memory, cognitive processing speed, attention, and executive functioning skills. Previous research has demonstrated that the aging process is not always necessitate a passive uncontrollable process. In fact, it is now understood that engaging in cognitive exercises can have generalized effects to one's mood, and emotional wellbeing. Brain plasticity remains responsive, even throughout one's older years of life. In addition, research has strongly supported that stimulating cortical areas associated with these functions will have generalized affects to one's auditory processing precision and speed, as measured by standardized instruments that 
are sensitive to subtle changes of these cognitive skill sets. Thus, one of the most effective ways that an individual can play a role in slowing cognitive decline is to continue actively exercising the brain throughout one's life.

Cognitive decline may be frightening for some individuals, especially if they do not have supportive familial relationships or access to adequate health care. In one's older years, cognitive functioning is directly correlated with safe, autonomous living. Older adults strive to remain "sharp" for as long as possible, in order to be able to effectively take care of themselves, and to remain independent. Therefore, it is understandable why this population would want to be aware of any intervention treatments that may help to promote the improved functioning of cognitive processes. Effective cognitive functioning is associated with independence, and thus improved quality of life.

Consequently, it is of clinical interest to investigate the effects of a unique therapeutic intervention treatment as a means of increasing cognitive functioning in this population. With a significant demand for evidence-based practice in the areas of maintaining the agility and strength of cognitive functions, research should focus on investigation of potential therapeutic techniques associated with targeting, and improving cognitive functioning. Specific, replicable intervention studies are needed in order to elucidate how cognitive based exercises can best be utilized when working with the geriatric population. Due to the arduous nature of conducting this kind of research, as it is requires an immense amount of effort from both the participant and examiners, the available body of research is limited. However, the results of the available research clearly indicate that there are significant relationships between cognitive exercise and subsequent improvement in cognitive functioning. The current study may add to the 
growing body of research that has already identified generalizable effects to global cognitive functioning in the elderly population, after engaging in cognitive training exercises.

\section{Plan of Study and Experimental Questions}

The purpose of the present study is to examine the efficacy of a specific cognitive skills training intervention with a group of 8 older adults, ages 70 and older, residing in an assisted living facility or a nursing home. The Five Task Approach (TFTA) will be implemented and analyzed. The novel approach consists of five cognitive stimulation exercises, or "tasks", that are strategically designed to target and improve cognitive processes associated with visual processing speed and working memory. It is our hopes that this intervention will also demonstrate sequential improvement of one's auditory processing skills. This study demonstrates that even brief cognitive intervention and exposure to tasks that may tax areas of the brain associated with processing and working memory can be altered, and strengthened, by participation in even a brief interaction with cognitive exercises. Thus, the following experimental questions will be answered:

1. Will TFTA participation show significant improvement on the five experimental tasks post-treatment intervention? The five tasks will include: "The Number Pairs Activity", "Flash Card Memory Drill”, "Sequential Number Memory (Reverse Order) Task", and "The Detail Picture Question Task." It is hypothesized that participant's performance will improve significantly on these five cognitive exercises, after the individual intervention.

2. Will participation in the TFTA result in a significant increase in mental status as measure by post-treatment administration on the Mini Mental State Exam (MMSE) 
(Folstein et al., 1975), relative to increased overall cognitive status? It is hypothesized that participant performance on the MMSE will improve on post treatment measurements, as global cognitive status may improve as a consequence of the TFTA intervention.

3. Will participation in the TFTA result in a significant increase in visual processing and visual perception as measured by performance on post-treatment administration on the Motor-Free Visual Perception Test-4 (MVPT-4) (Colarusso \& Hammill, 2015)? It is assumed that participant performance on the MVPT-4 will improve significantly on post intervention measurements. It is our expectation that the TFTA intervention may increase overall processing speed, thereby resulting in improved scores on the MVPT-4.

4. Will participation in the TFTA result in a significant increase in cognitive status (i.e., working memory, executive functioning, and attention) as measured by performance on post-treatment administration for the Dementia Rating Scale-2 (DRS-2) (Jurica, Leitten, \& Mattis, 2001)? It is hypothesized that participant performance on the DRS-2 will improve significantly on post intervention measurements. It is theorized that engaging in the TFTA will enhance one's cognitive agility, and may have generalized affects to cognitive skills that were not directly targeted during intervention (attention, initiation and perseveration, and conceptualization). 


\section{CHAPTER II}

\section{METHOD}

\section{Participants}

Study participants included 16 monolingual fluent English-speaking individuals ages $70+$ years of age. Each participant was randomly assigned to either the treatment or the control group. All participants live in an assisted living community or a nursing home, located within Broward or Palm Beach County, in South Florida. All participants possessed a minimum of a High School diploma. If an individual was color blind (asked via self-report, and verified via quick clinical screening), or if they could not read size 20 or 30 font clearly, even when using appropriate corrective vision apparatuses, they were excluded from the study. If the participant failed a modified hearing screening for older adults (Mulrow \& Lichtenstein, 1991), he or she was excluded from the study.

All participants were cognitively alert, and oriented to place and purpose. All participants received a minimum score of 25 on the Mini Mental State Exam (MMSE) (Folstein et al., 1975). In addition, all participants scored within 1 standard deviation of the mean on the Motor-Free Visual Perception Test-4 (MVPT- 4) (Colarusso \& Hammill, 2015). No participant had sustained a traumatic brain injury (TBI) in the last 12 months. No participant was included if they had been diagnosed with a neurological disorder known to moderately to severely affect cognition. Participants were excluded if they presented with delirium, confusion, and/or other obvious disturbances in consciousness and orientation. Participants were not considered for inclusion in the study if they were currently receiving speech and language services from another provider or if they were 
currently participating in another research study. All of the aforementioned criteria, in addition to on-going clinical judgment, and visual screeners was met in order for the 16 participants to remain appropriate for participation utilizing the TFTA brain training intervention.

Potential participants were recruited for the study by flyers placed strategically throughout various facilities in which they dwell. In addition, individuals were informed of the experiment via word of mouth, from employees, family members, and caregivers. Participants were recruited via a randomized convenience sample. As indicated, a total of 16 residents were randomly selected for study participation. Independent t-tests on age and education between the two groups indicate that there was no significant difference between the groups relative to age $(p>.05)$ or education level $(p>.05)$. 
Table 1. Participant Demographics

\begin{tabular}{|c|c|c|c|}
\hline ACTIVE & GENDER & AGE & $\begin{array}{c}\text { EDUCATION } \\
\text { (in years) }\end{array}$ \\
\hline $\mathbf{1}$ & $\mathrm{F}$ & 89 & 12 \\
\hline $\mathbf{2}$ & $\mathrm{F}$ & 76 & 12 \\
\hline 3 & $\mathrm{~F}$ & 94 & 18 \\
\hline $\mathbf{4}$ & $\mathrm{F}$ & 81 & 14 \\
\hline 5 & $\mathrm{M}$ & 83 & 19 \\
\hline $\mathbf{6}$ & $\mathrm{F}$ & 75 & 14 \\
\hline 7 & $\mathrm{M}$ & 77 & 12 \\
\hline $\mathbf{8}$ & $\mathrm{F}$ & 86 & 12 \\
\hline Mean & $2 \mathrm{M}, 6 \mathrm{~F}$ & 82.63 & 14.13 \\
\hline SD & & 6.74 & 2.85 \\
\hline Range & & $75-94$ & $12--19$ \\
\hline
\end{tabular}

\begin{tabular}{|c|c|c|c|}
\hline CONTROL & GENDER & AGE & $\begin{array}{c}\text { EDUCATION } \\
\text { (in years) }\end{array}$ \\
\hline $\mathbf{1}$ & $\mathrm{F}$ & 80 & 18 \\
\hline 2 & $\mathrm{~F}$ & 90 & 16 \\
\hline 3 & $\mathrm{~F}$ & 91 & 12 \\
\hline $\mathbf{4}$ & $\mathrm{M}$ & 85 & 14 \\
\hline 5 & $\mathrm{M}$ & 86 & 12 \\
\hline $\mathbf{6}$ & $\mathrm{F}$ & 90 & 16 \\
\hline 7 & $\mathrm{~F}$ & 85 & 12 \\
\hline $\mathbf{8}$ & $\mathrm{M}$ & 82 & 12 \\
\hline Mean & $3 \mathrm{M}, 5 \mathrm{~F}$ & 86.13 & 14 \\
\hline SD & & 3.98 & 2.39 \\
\hline Range & & $80-91$ & $12--18$ \\
\hline
\end{tabular}

\section{Pre-Experimental Testing}

All participants were administered the Mini Mental State Exam (MMSE)

(Folstein et al., 1975), the Motor-Free Visual Perception Test-4 (MVPT-4) (Colarusso \& 
Hammill, 2015) and the Dementia Rating Scale-2 (DRS-2), prior to and following the completion of the intervention. Performance and responses on these measures were recorded in order to compare scores, and to allow examiners to draw conclusions in regards to whether results of this treatment generalized to cognitive skills not targeting during intervention. Scores obtained on pre-experimental measures served as a baseline for experimental treatment participants.

Each participant underwent a brief hearing screening. A modified hearing screening for older adults was conducted using a standard audiometer at $40 \mathrm{~dB}$ HL through the speech frequencies, $1000 \mathrm{~Hz}, 2000 \mathrm{~Hz}$, and $4000 \mathrm{~Hz}$ (Mulrow \& Lichtenstein, 1991). Individuals who did not meet the hearing thresholds were not included in the study.

The participants' vision was screened via the "Visual Screener/Picture Naming Task" (Appendix B), which consists of 5 capitalized words, font size 20, Times New Roman. All participants demonstrated ability to correctly label and identify the colors of all of the 5 colored pictures, measuring $2 \times 2$ inches. If a participant was not able to describe all 5 pictures accurately, including accurate color description, they were excused from the study. The examiner briefly administered this screener before the start of every session to control for any visual changes. 


\section{General Information}

The current study has been approved by the Institutional Review Board at Florida International University. Informed Consent was provided via a written format (Appendix C). Treatment intervention took place within the facility where the participants live, in a quiet location of his or her preference, typically the resident's apartment. The clinician sat across from the participant. The examiner was cognizant of the fact that the participants were volunteering his or her time, and energy, to participate in this study. The exact purpose of the study was not disclosed to any of the participants; however, all participants were informed that they were playing "brain games", and participating in measures to evaluate their skills, with the hopes of potentially increasing cognitive functioning skills, including memory and processing speed. No reward of any kind was offered and there were no additional incentives presented as to coax participation. Treatment participants demonstrated an understanding that this intervention may or may not increase cognitive functioning skills, but that participation would inflect no negative side effects.

The cognitive intervention for each of the 8 active treatment participants included an initial and final session in which the 3 standardized batteries were administered, and scores recorded. The initial and final sessions took approximately 60 minutes each. In addition, the participants were administered a baseline performance measure of the TFTA, without therapeutic cueing; this session took approximately 30 minutes. The participants were then given therapeutic cueing during the following 4, 30-minute, individual treatment sessions. The active treatment participants met with the investigator for a total of 7 sessions. The examiner was diligent about time management, adhering to 
the specific time constraints of each activity, as each session lasted no longer than 35 minutes. A baseline measure of performance on the TFTA was taken before therapeutic intervention is introduced. Each participant was seen for a minimum of 2 to 6 sessions per week, over the course of 2-4 consecutive weeks, with a maximum of one session per day. After initial interaction with a participant, no participant went longer than 7 days without receiving intervention, or follow up testing, unless they were dismissed from the study. All of the 5 intervention games were administered in their entirety in order for the session to be considered complete and valid. If the participant was unable to complete an activity, or needed to stop for more than 10 minutes at any point throughout the session, for whatever reason, that session was not counted, and another session was scheduled for that week.

The control group met all of the inclusionary and exclusionary factors. They were similarly administered the MMSE (Folstein et al., 1975), the MVPT-4 (Colarusso \& Hammill, 2015), and the DRS-2 (Jurica et al., 2001). Control participants participated in 4 sessions of game activities, including the card game "War", "Tic-Tac-Toe, and "Hangman" with the investigator (See Appendix L for Control Condition Protocol). Each of the control activities were explained to the participant, as presented, if they were not already familiar with the games. The control condition did not have any interaction with the "The Five Task Approach", or the therapeutic cues. Initial and final testing was conducted on two separate days, utilizing the 3 standardized batteries. Similarly, the 2 sessions took approximately 60 minutes each. In addition, those participants in the control group met for 4, 25 to 30 minute sessions. Thus, the control participants met 6 times, 2 times for pre/post testing, and 4 times for the 3 game activities. If the participant 
needed to stop for more than 10 minutes at any point throughout the session, for whatever reason, that session was not be counted, and another session was scheduled for that week. The scores obtained from the control group on the standardized measures were compared to the scores obtained from participants in the treatment condition both pre and post intervention, as well as against their own pre-activity scores.

\section{Experimental Testing}

\section{Screening:}

After fulfilling all inclusionary requirements, treatment participants were screened by completing the "Visual Screener/Picture Naming Task", which consists of 5 capitalized words, font size 20, Times New Roman. The participant was also required to correctly label all 5 colored pictures, measuring $2 \times 2$ inches. If a participant was not able to label, and describe all 5 pictures correctly, they were excused from the study. The examiner briefly administered this screener before the start of every session to control for visual changes (Appendix B). If a participant failed to earn a score of $100 \%$ on the "Visual Screener/Picture Naming Task", they were immediately excluded from the study. The investigator recorded responses of the 5 "Pre-Session Questions" (see Appendix D) prior to each of the 5 sessions. These questions included whether or not the participant had stopped or started any new medication since the last session, if they had sustained any injuries, if there were any significant changes in daily activity, confirmation that they were able to comfortably see the stimuli, and a 1-5 Likert scale question in regard to overall well-being that day. 


\section{Materials:}

For the implementation of the experimental intervention, different materials were required for each of the 5 activities. Detailed protocols for each of the 5 cognitive braingame activities, known as "The 5 Task Approach", are included in Appendices G-K, along with associated scoring sheets, and answer keys. The materials required included all protocols, a pen/pencil, a stopwatch, the 50 flashcards consisting of concrete word items printed in size 20 font, Times New Roman, and the 50 colored images each on a separate page. The investigator had access to all of the protocols, and necessary visual stimuli required to "play" each brain-game before initiation of each session. In addition, the investigator had access to the associated record and scoring sheets, along with a thorough understanding of how to utilize the specific cues for each activity. All scores were recorded for each of the five tasks. See Appendix M for baseline and record forms.

For interaction with the control group, a deck of 52 jumbo-sized text playing cards, paper and pen/pencil, and a dry erase board/dry erase pen (optional) was required. See Appendix L for control condition protocol.

\section{Experimental Procedures/Protocols:}

The investigator fluently explained all procedures of the TFTA and ensured that the participant's fully comprehended all of the instructions provided. This was confirmed by asking each participant to verbally recall and reiterate all of the "rules", and cues to be implemented, prior to beginning a brain-game. Each participant's baseline scores were obtained via the aforementioned batteries, as well as by completing each of the 5 activities in "The Five Task Approach", without any cueing for the first session. Prior to 
initiation of and administration of the TFTA, the investigator thoroughly familiarized themselves with the 5 protocols, all materials required, and the unique target cues to be employed within each activity. The investigator understood how to utilize the specified cues appropriately, as well as how to implement them appropriately, while ensuring that each was utilized a minimum of one time. The five tasks included: "The Number Pairs Activity", "Flash Card Memory Drill", "Sequential Number Memory (Reverse Order) Task", "The Letter Circle Task", and "The Detail Picture Question Task."

\section{The Number Pairs Activity (Appendix G) consisted of the participant using his or} her visual processing abilities to identify matches as quickly as possible. The investigator presented the participant with 10 rows of numbers, each containing 10 double-digit numbers. The numbers were printed in size 30, Times New Roman font. The rows measured $5^{\prime}$ by $2.5^{\prime}$. Each row contained a number pair. The participant were instructed to find and circle the pairs as quickly, and as accurately, as possible, within 1 minute.

2. The Flash Card Memory Drill (Appendix H) activity consisted of the participant being presented with 10 flashcards consisting of 10 different unrelated words of concrete objects. The investigator read the card as they visually presented it to the participant and placed it on the table in front of them. After the 10 cards had been presented, the investigator removed the cards, and asked the participant to recall as many of the words as possible, within 2 minutes.

3. The Sequential Number Memory (Reverse Order) task (Appendix I) consisted of the participant recalling 10 sets of 4 numbers presented orally, in reverse order. Each set of 4 numbers was presented with a 2 second delay. The participant had a maximum of 30 
seconds to recall the 4 numbers in reverse order.

4. The Letter Circle Task (Appendix J) consisted of the participant visually searching a sheet filled with 60 letters, with the goal of identifying and circling 10 repeated letters, within 1 minute. The letter to be identified and circled was verbally given to the participant right before the start of the search. Participants had 1 minute to find and circle all of the 10 repeated letters.

5. The Detail Picture Question Task (Appendix K) consisted of the investigator presenting the participant with an image for 10 consecutive seconds. After the 10 seconds, the image was removed and the participant was asked a specific question regarding the image. All question types were able to answered correctly in 1 word. Participants had 10 seconds to provide an answer.

Participant's scores were measured as a percentage correct out of the total possible correct answers specified on the protocols (100\%). All response times, percentages, and cues utilized, were recorded after the completion of each activity, during each of the 4 sessions. The first interaction with the TFTA served as the individual's baseline. The proceeding 4 sessions consisted of the use of interventional cueing. Quantitative data obtained from the $5^{\text {th }}$ interaction with the TFTA served as the individual's post scores.

\section{Interventional Cueing}

Interventional cueing was implemented by following the specific, operationalized instructions provided in each for each of the 5 protocols, as well as in the Explanation of Cues, and Participant Cue Script (see Appendix E and F). Therapeutic cues implemented 
during therapy sessions 2 through 4 utilizing The Five Task Approach employed 3 main principles, the concept of a "visual mental screen", "information chunking", and "word associations/cognitive chaining."

The concept of a "visual mental screen" can best be explained as a "mental dry erase board." Individuals may utilize this "mental screen" by visualizing/projecting both visual and auditory information on to this "screen." It will appear to be a foreign concept to many participants; therefore, the investigator must explain that this mental screen is completely controlled by them. The investigator will tell the participants to imagine the screen as if it is "their mind's eye.". They may ask the participants to verbalize the color, shape, and size that he or she has chosen for their unique mental screen. The investigator may have the participant practice visualizing items on his or her screen before intervention begins. For example, they may have the participants practice varying the color of the screen on command as a means of practicing volitional control of the images they choose to project on to it. Further, the investigator may ask the participant to visualize images such as common objects (apple, car, dog) on his or her mental screen, and have them assign details to the images. Utilization of the visual mental screen will assist participants by providing them with a means of visualizing information in his or her mind, and thereby providing them with a secondary source of a memory or presented stimuli.

The concept of information "chunking" can best be explained as the grouping of information, while ideally simultaneously visualizing the stimuli on one's visual mental screen. One may choose to "chunk" information in groups of 2 or more items depending on the content of the presented information. In essence, chunking is a means of 
organizing presented stimuli in to smaller, and more manageable groups of information. Again, this process is unique to every individual. The investigator will explain that this process is one that is different and most effective for each individual in a different way. The investigator will attempt to assist the participant in a comprehensive understanding of this concept. Consider the following example: If a participant hears the numbers " $2,1,4,3$ ", the investigator will instruct the participant to visualize the numbers 2 and 1 on one side of their screen, and the numbers 4 and 3 on the other side. Other individuals may prefer to chunk the middle 2 numbers and have their screen read 2 independently on the left, with 1 and 4 chunked in the middle, with 3 independently on the right of the screen.

Chunking will assist the participant by providing them with a means of visualizing, and organizing, information in his or her mind. Another example may be provided in terms of organizing visual stimuli; that is, if the participant is looking at an image of 7 people standing on a pier, the investigator may encourage the participant to group the individuals into groups of 3 with one odd man out. Perhaps that participant may utilize chunking more effectively by making a group of 4 and a group of 3 individuals in his or her mind. The participant may even opt to mentally underline the middle individual, and chunk the other 6 in to 2 groups of 3 . The investigator will make sure that the participant is clear on this concept before initiation of the second treatment session. The use of information chunking will assist participants by providing participants with a means of organizing the information that is presented to them, as opposed to becoming overwhelmed and ignoring details that they could have retained.

The use of information chaining and association can best be explained as the 
process by which individuals turn presented information in to terms that are unique, personal, and meaningful to them. For example, when an individual is presented with auditory information, they internalize the information by coming up with a story line that connects the words or information to be remembered. An example of this is an individual trying to remember 3 unrelated words: "dog, truck, grass." He or she may come up with a silly or unique picture that they come up with and visualize on his or her mental screen. The individual could remember those 3 words by visualizing a big dog driving a small truck on a lawn of freshly cut grass. The story will develop as the words are presented. If there were more items to be remembered, they could continue on with this cognitive image, and develop additional ideas to facilitate memory of the stimulus items. It is thought that the sillier the "story" or image, the harder it will be to forget, and thus the easier it will be to recall.

Another example would be if an individual is trying to remember a string of numbers, he or she may utilize their mental screen, in addition to chunking this information, in order to make the numbers unique and meaningful to the, as a means of aiding with future recall. Perhaps the numbers presented are representative of a meaningful date to that participant, or perhaps if chunked, the numbers are indicative of his or her apartment number. These types of associations should be pointed out to the participant. The investigator will make sure that the participant is clear on this concept before initiation of the second treatment session. It is assumed that these associations will facilitate one's recall by providing them with a secondary, individualized memory to rely on when attempting to recall information. The use of cognitive associations will assist 
participants by providing participants with a means of organizing the information that is presented to them in a unique way that will ideally aid in creating a secondary memory.

These therapeutic cues were thoroughly explained to each of the participants, and reiterated before the beginning of sessions 2 through 4 . Other clinical cues involved the investigator providing the participants with positive, verbal encouragement. The investigator encouraged participants to keep trying even if they were not performing well, as well as to remain focused. In addition, the investigator encouraged participants to work as quickly as possible during the activities. For appropriate tasks, the clinician cued the participant to utilize auditory rehearsal (reiterating information silently to one's self), as well as to utilize a horizontal visual search style when presented stimuli. If the participant appeared to approach the activities with a disorganized style, and was overtly not implementing cues, the investigator reminded the participant of the cues. The investigator provided participants with a timing cue, when $50 \%$ of the delineated time was left. That is, on task 1 participants were informed when they had 30 seconds remaining. For task 2, participants were informed when there was 1-minute remaining. On task 3, participants were alerted when they had 15 seconds remaining, followed by an alert for task 4 , at 30 seconds. Finally, participants were altered on task 5 when there was 5 seconds remaining to provide a response. The aforementioned clinical cues were assumed to cause participants to demonstrate improvement on post testing measures of the tasks, as well as improve the approach an individual will implement when attempting to complete a task in the future.

Again, no cueing occurred during the baseline measurements of performance on each of the five activities. In order to ensure that participants utilized the cues effectively, 
the investigator explained each of the strategies before initiation of the five tasks. The examiner explained to each participant that all of the cues should be remembered, and utilized in subsequent therapy sessions, and that they can be used to maximize cognitive growth not only during therapy, but also in the future when engaging in functional activities of daily living. It was also reiterated to the participants that these cues can, and should, be used in combination with one another, as each cue builds on one another. Clinicians will keep a separate folder, containing the continuous performance data for each of the participants.

\section{Design and Data Analysis}

The present investigation implemented a within subjects, quantitative treatment study. The study recruited performance-based, criterion as well as standardized measures, analyzed utilizing a pre/post-test design. Related t-tests were used to analyze group data for the TFTA intervention cues pre and post therapeutic cueing. In addition, related t-test analyses were used to examine pre and post treatment performance differences on mental status, as measured by the Mini Mental State Exam, global cognitive status and working memory, via the Dementia Rating Scale-2, and overall processing speed, as measured by the Motor-Free Visual Perception Test-4. Independent t-tests were used to compare performance of the control and the treatment groups on the standardized measures, both pre and post-treatment. 


\section{CHAPTER III}

\section{RESULTS}

The purpose of this study was to examine the effects of a brief cognitive intervention (The TFTA) on various aspects of cognitive functioning with the geriatric population. 16 participants were recruited for participation in this study. Of the 16,8 participants were randomly selected to participate in the TFTA intervention, for 5, 30-minute sessions. The 8 control participants were not exposed to the TFTA intervention, and instead played games of War, Tic-Tac-Toe, and Hangman, on 4 separate occasions, for 30 minutes, with the investigator. All 16 participants were administered The Dementia Rating Scale-2, The Motor Free Visual Perception Test-4, as well as The Mini Mental State Examination before and after intervention. In order to conduct analyses between, and within groups, independent and related t-tests were conducted and analyzed.

The first experimental question addressed whether therapeutic intervention and cueing, would have a significant effect on post intervention performance on the 5 TFTA tasks when compared to baseline scores taken prior to the initiation of cues. To answer this question, pre-treatment/baseline performance was compared to post-treatment performance for all 5 experimental tasks for the 8 participants in the active group. These data are presented in Table 2. Related t-tests were conducted on these data for each of the experimental tasks. Please refer to Figures 1 through 5 for a visual display of each of the 8 participant's overall performance across the 5 sessions, on each of the 5 tasks respectively. 
For The Number Pairs Task, a statistically significant difference/improvement was noted on post-test administration, when compared to baseline measures for the active group ( $\mathrm{p}<.005$ ); refer to Figure 1 for all active participant scores across the 5 sessions.

Analysis of The Flash Card Memory Drill data revealed a highly significant difference between baseline and post testing performance ( $p<.009)$. Figure 2 displays all active participant scores across the 5 sessions.

For the third task, Sequential Number Memory (Reverse Order), related t-test analysis revealed a highly significant difference between baseline and post treatment sessions $(\mathrm{p}<.0002)$. Figure 3 is a display of all active participant scores across the 5 sessions for this task.

For the Letter Circle Task, related t-test analysis did not reveal a significant difference between baseline and post treatment scores ( $p>.05)$. Figure 4 is a display of all active participant scores across the 5 task sessions.

Analysis of data for the Detailed Picture task revealed a highly significant difference in performance between baseline and post treatment sessions scores $(\mathrm{p}<.009)$ for the active group. Figure 5 is a display of all active participant scores across the 5 sessions for this task.

Appendix $\mathrm{N}$ includes the data for each of the 5 session scores, for each of the 5 TFTA tasks, for all 8 active participants. 
Table 2. Baseline and Post-Treatment Data for the 5 Experimental Tasks

\begin{tabular}{|c|c|c|c|c|c|c|}
\hline $\mathbf{N}$ & Session & $\begin{array}{l}\text { Number } \\
\text { Pairs } \\
\text { Task }\end{array}$ & $\begin{array}{l}\text { Flash } \\
\text { Card } \\
\text { Memory } \\
\text { Drill } \\
\end{array}$ & $\begin{array}{l}\text { Sequential } \\
\text { Number } \\
\text { Memory } \\
\text { (Reverse) }\end{array}$ & $\begin{array}{l}\text { Letter } \\
\text { Circle } \\
\text { Task }\end{array}$ & $\begin{array}{l}\text { Detailed } \\
\text { Picture } \\
\text { Question } \\
\text { Task } \\
\end{array}$ \\
\hline \multirow[t]{2}{*}{1} & PRE & 50 & 60 & 30 & 100 & 50 \\
\hline & POST & 90 & 70 & 60 & 100 & 70 \\
\hline \multirow[t]{2}{*}{2} & PRE & 60 & 50 & 20 & 100 & 60 \\
\hline & POST & 100 & 80 & 40 & 100 & 100 \\
\hline \multirow[t]{2}{*}{3} & PRE & 70 & 30 & 10 & 90 & 60 \\
\hline & POST & 70 & 70 & 30 & 100 & 60 \\
\hline \multirow[t]{2}{*}{4} & PRE & 70 & 40 & 60 & 100 & 70 \\
\hline & POST & 90 & 90 & 80 & 100 & 80 \\
\hline \multirow[t]{2}{*}{5} & PRE & 40 & 50 & 30 & 80 & 60 \\
\hline & POST & 50 & 70 & 50 & 100 & 70 \\
\hline \multirow[t]{2}{*}{6} & PRE & 80 & 80 & 40 & 100 & 40 \\
\hline & POST & 100 & 100 & 80 & 100 & 90 \\
\hline \multirow[t]{2}{*}{7} & PRE & 70 & 80 & 20 & 100 & 50 \\
\hline & POST & 100 & 70 & 30 & 100 & 70 \\
\hline \multirow[t]{2}{*}{8} & PRE & 50 & 60 & 10 & 90 & 50 \\
\hline & POST & 60 & 90 & 50 & 100 & 70 \\
\hline \multirow[t]{2}{*}{ MEAN } & PRE & 61.25 & 56.25 & 27.5 & 95 & 55 \\
\hline & POST & 82.5 & 80 & 52.5 & 100 & 76.25 \\
\hline \multirow[t]{2}{*}{ SD } & PRE & 13.56 & 17.68 & 16.69 & 7.56 & 9.26 \\
\hline & POST & 19.82 & 11.95 & 19.82 & 0 & 13.02 \\
\hline \multirow[t]{2}{*}{ RANGE } & PRE & $40-80$ & $30-80$ & $10-60$ & $80-100$ & $40-70$ \\
\hline & POST & $50-100$ & $70-100$ & $30-80$ & 100 & $60-100$ \\
\hline
\end{tabular}




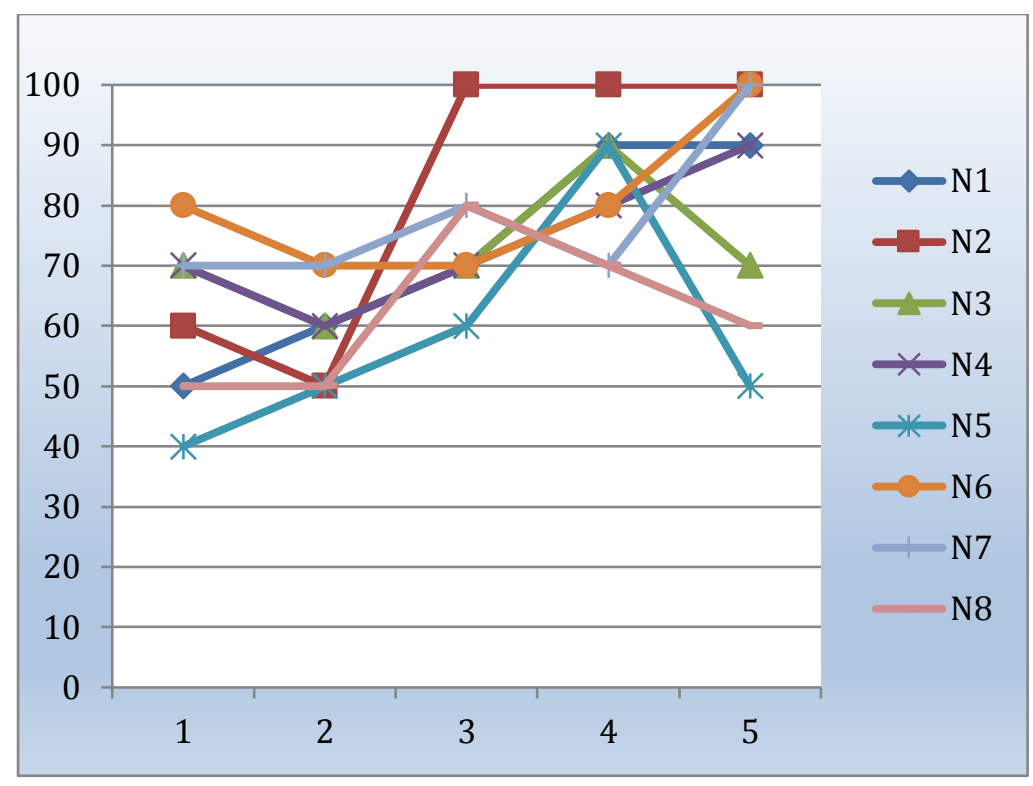

Figure 1. Active Participant Performance on The Number Pairs Activity Task

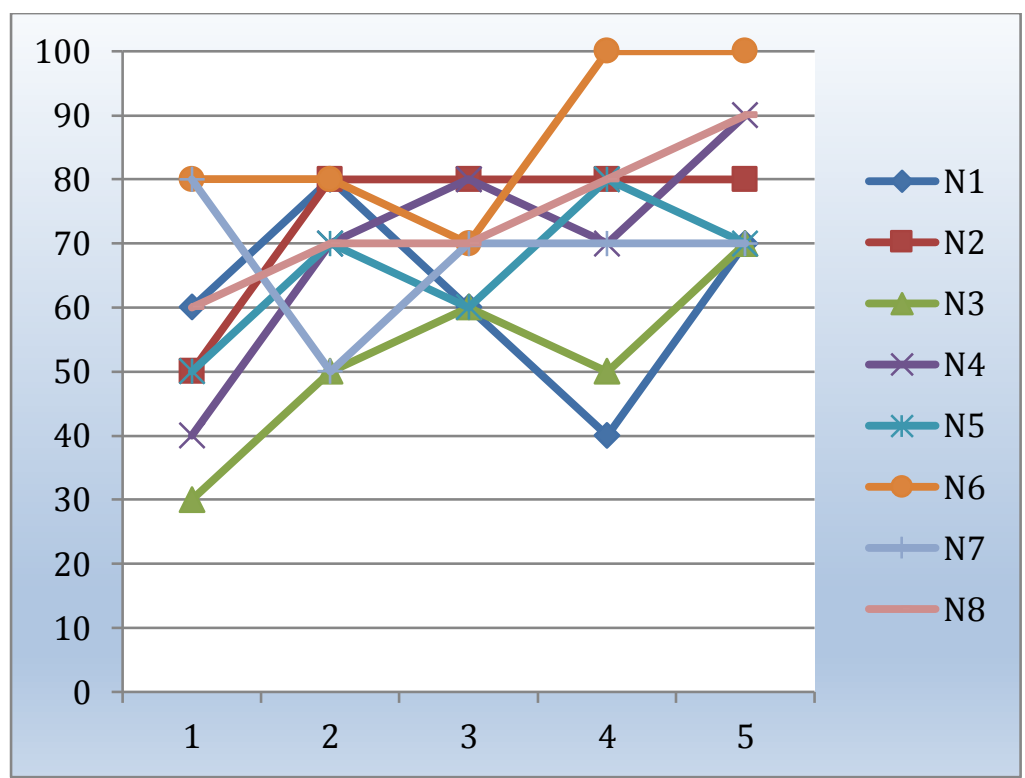

Figure 2. Active Participant Performance on The Flash Card Memory Drill Task 


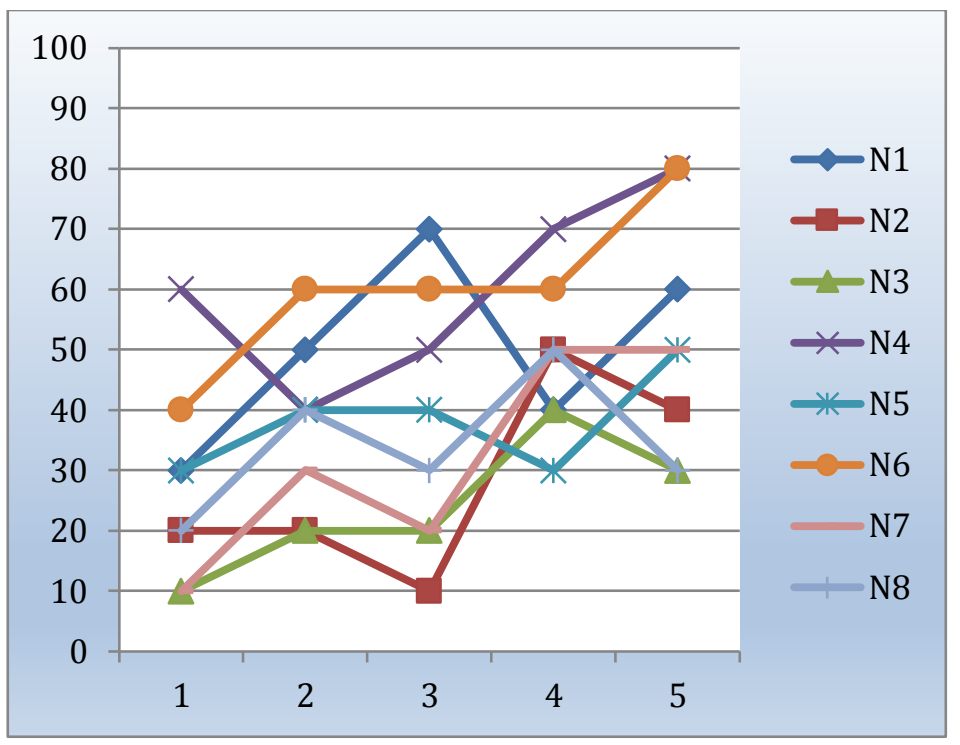

Figure 3. Active Participant Performance on The Sequential Number Memory (Reverse Order) Task

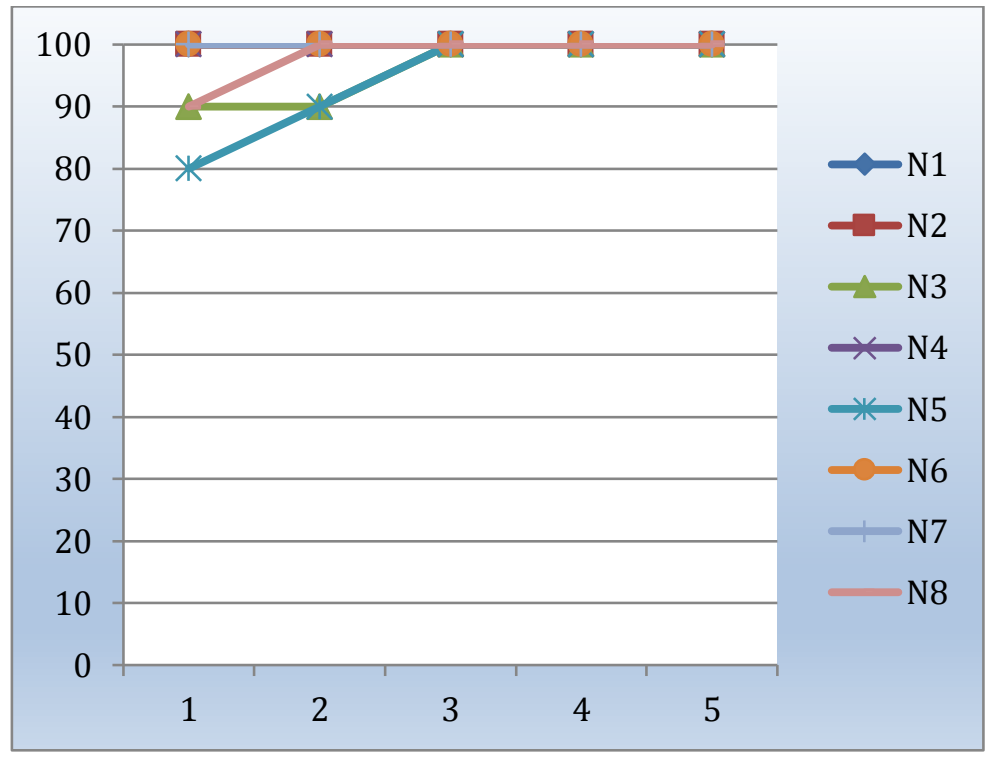

Figure 4. Active Participant Performance on The Letter Circle Task 


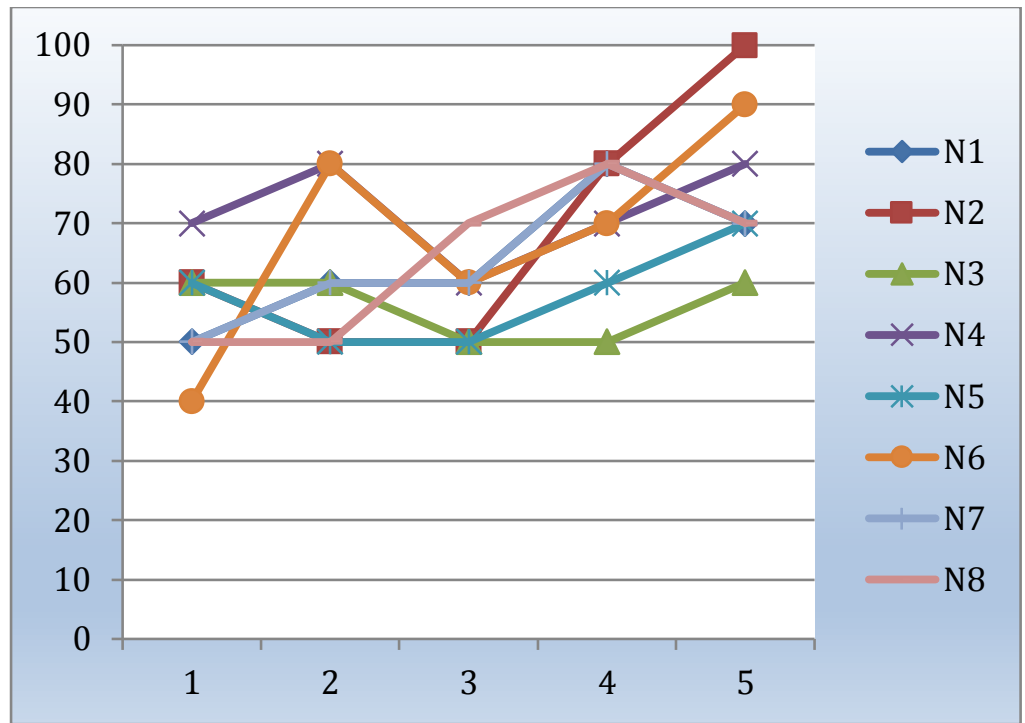

Figure 5. Active Participant Performance on The Detailed Picture Question Task 
The second experimental question addressed whether the TFTA intervention revealed a significant difference in performance on The Dementia Rating Scale-2 (DRS2) post-treatment, thus increasing participant's global cognitive functioning, including generalized skills associated with executive functioning (attention, initiation/perseveration, and conceptualization). The baseline and post-treatment DRS-2 data for both groups are presented in Table 3. Difference scores were calculated between pre- and post-testing data for comparison between the two groups. An independent t-test between groups on the difference scores revealed no significant difference between the groups on pre and post-test performance $(\mathrm{p}>.05)$. However, related t-tests conducted on the pre and post-treatment administration of the DRS-2 separately for the active and control groups revealed a highly significant difference $(\mathrm{p}<.007)$ between the baseline and post intervention scores in terms of generalization of improved cognitive functioning for the active group. There was no significant difference found for the control group upon comparison of baseline and post-test scores on the DRS-2 ( $p>.05)$. 
Table 3. The Dementia Rating Scale-2 Pre/Post Scores

\begin{tabular}{|c|c|c|c|c|}
\hline \multicolumn{2}{|c|}{$\begin{array}{l}\text { The Dementia } \\
\text { Rating Scale-2 }\end{array}$} & \multirow[t]{2}{*}{$\mathbf{N}$} & \multirow[t]{2}{*}{$\begin{array}{l}\text { Scaled } \\
\text { Score } \\
\text { (Pre) }\end{array}$} & \multirow[t]{2}{*}{$\begin{array}{l}\text { Scaled } \\
\text { Score } \\
\text { (Post) }\end{array}$} \\
\hline Active & & & & \\
\hline & & 1 & 12 & 15 \\
\hline & & 2 & 9 & 11 \\
\hline & & 3 & 8 & 11 \\
\hline & & 4 & 8 & 13 \\
\hline & & 5 & 6 & 10 \\
\hline & & 6 & 13 & 13 \\
\hline & & 7 & 10 & 10 \\
\hline & & 8 & 8 & 10 \\
\hline & Mean & & 9.25 & 11.625 \\
\hline & SD & & 2.31 & 1.85 \\
\hline & Range & & $6--3$ & $10--15$ \\
\hline
\end{tabular}

\begin{tabular}{|l|r|r|r|}
\hline & N & $\begin{array}{l}\text { Scaled } \\
\text { Score } \\
\text { (Pre) }\end{array}$ & $\begin{array}{l}\text { Scaled } \\
\text { Score } \\
\text { (Post) }\end{array}$ \\
\hline & 1 & 9 & 12 \\
\hline & 2 & 7 & 9 \\
\hline & 3 & 7 & 10 \\
\hline & 4 & 8 & 12 \\
\hline & 5 & 10 & 11 \\
\hline & 6 & 11 & 12 \\
\hline & 7 & 13 & 13 \\
\hline & 8 & 11 & 12 \\
\hline Mean & & 9.5 & 11.375 \\
\hline SD & & 2.14 & 1.30 \\
\hline Range & & $7-13$ & $9--13$ \\
\hline
\end{tabular}


The third experimental question addressed whether the TFTA intervention revealed a significant difference in performance on The Motor Free Visual Perception Test-4 (MVPT-4), thus improving participant's visual processing abilities post intervention. The baseline and post-treatment MVPT-4 data for both groups are presented in Table 4. Difference scores were calculated between pre and post-testing data for comparison between the two groups. An independent t-test between groups on the difference scores revealed a highly significant difference between the groups on pre and post-test performance $(\mathrm{p}<.001)$. Furthermore, related t-tests conducted on the pre and post-treatment administration of the MVPT-4 separately for the active and control groups revealed a highly statistically significant difference in performance on pre and postadministration of the MVPT-4 ( $<<.0005)$ for the active group with no significant difference for the control group(p>.05). 
Table 4. The Motor-Free Visual Perception Test-4 Pre/Post Scores

\begin{tabular}{|l|r|r|r|}
\hline \multicolumn{4}{|l|}{$\begin{array}{l}\text { The Motor-Free Visual } \\
\text { Perception Test-4 }\end{array}$} \\
\hline Active & N & $\begin{array}{l}\text { Standard } \\
\text { Score } \\
\text { (Pre) }\end{array}$ & $\begin{array}{l}\text { Standard } \\
\text { Score } \\
\text { (Post) }\end{array}$ \\
\hline & & & 129 \\
\hline & 1 & 106 & 113 \\
\hline & 2 & 105 & 113 \\
\hline & 3 & 98 & 109 \\
\hline & 4 & 88 & 100 \\
\hline & 5 & 88 & 110 \\
\hline & 6 & 89 & 100 \\
\hline & 7 & 96 & 108 \\
\hline & 8 & 96 & 110.25 \\
\hline Mean & & 95.75 & 9.13 \\
\hline SD & & 7.19 & $100-129$ \\
\hline Range & & $88-106$ & \\
\hline
\end{tabular}

\begin{tabular}{|l|r|r|r|}
\hline Control & $\mathbf{N}$ & $\begin{array}{l}\text { Standard } \\
\text { Score } \\
\text { (Pre) }\end{array}$ & $\begin{array}{l}\text { Standard } \\
\text { Score } \\
\text { (Post) }\end{array}$ \\
\hline & & & \\
\hline & & & 96 \\
\hline & 1 & 85 & 100 \\
\hline & 2 & 96 & 104 \\
\hline & 3 & 98 & 96 \\
\hline & 4 & 100 & 100 \\
\hline & 5 & 108 & 100 \\
\hline & 6 & 94 & 102 \\
\hline & 7 & 102 & 86 \\
\hline & 8 & 90 & 98 \\
\hline Mean & & 96.625 & 5.55 \\
\hline SD & & 7.15 & $86-104$ \\
\hline Range & & $85-108$ & \\
\hline
\end{tabular}

The last experimental question addressed whether the TFTA intervention revealed a significant difference in performance on The Mini Mental State Examination (MMSE), thus improving participant's global cognitive status post intervention. The baseline and post-treatment MMSE data for both groups are presented in Table 5. Difference scores were calculated between pre- and post-testing data for comparison between the two groups. An independent t-test between groups on the difference scores revealed no significant difference between the groups on pre and post-test performance ( $p>.05)$.

However, separate related t-tests conducted on the pre and post-treatment administration of the MMSE separately for the active and control groups revealed statistically significant differences $(\mathrm{p}<.03)$ between testing for both groups. 
Table 5. The Mini Mental State Exam Pre/Post Scores

\begin{tabular}{|l|r|r|r|}
\hline $\begin{array}{l}\text { The Mini } \\
\text { Mental State } \\
\text { Examination } \\
\text { (MMSE) }\end{array}$ & N & $\begin{array}{l}\text { Raw } \\
\text { Score } \\
\text { (Pre)* }\end{array}$ & $\begin{array}{l}\text { Raw } \\
\text { Score } \\
\text { (Post)* }\end{array}$ \\
\hline Active & & & \\
\hline & 1 & 28 & 29 \\
\hline & 2 & 29 & 30 \\
\hline & 3 & 25 & 29 \\
\hline & 4 & 25 & 30 \\
\hline & 5 & 27 & 27 \\
\hline & 6 & 30 & 30 \\
\hline & 7 & 28 & 30 \\
\hline & 8 & 28 & 30 \\
\hline Mean & & 27.5 & 29.375 \\
\hline SD & & 1.77 & 1.06 \\
\hline Range & & & \multicolumn{2}{|l}{} \\
& & $25--30$ & $27--30$ \\
\hline
\end{tabular}

\begin{tabular}{|c|c|c|c|}
\hline & $\mathbf{N}$ & $\begin{array}{l}\text { Raw } \\
\text { Score } \\
\text { (Pre)* }\end{array}$ & $\begin{array}{l}\text { Raw } \\
\text { Score } \\
\text { (Post)* }\end{array}$ \\
\hline \multicolumn{4}{|l|}{ Control } \\
\hline & 1 & 24 & 26 \\
\hline & 2 & 28 & 30 \\
\hline & 3 & 25 & 27 \\
\hline & 4 & 27 & 30 \\
\hline & 5 & 26 & 26 \\
\hline & 6 & 30 & 29 \\
\hline & 7 & 29 & 30 \\
\hline & 8 & 26 & 28 \\
\hline Mean & & 26.875 & 28.25 \\
\hline SD & & 2.03 & 1.75 \\
\hline Range & & $24--30$ & $26-30$ \\
\hline \multicolumn{2}{|c|}{$\begin{array}{l}* \text { MMSE } \\
\text { max raw } \\
\text { score is } 30 .\end{array}$} & & \\
\hline
\end{tabular}




\section{CHAPTER IV}

\section{DISCUSSION}

The purpose of this study was to evaluate the effects of the TFTA intervention within an active group of 8 geriatric participants pre and post therapeutic cueing. The 8 randomly selected individuals participated in the intervention for 5, 30-minute sessions which included five cognitive brain games: Numbers Pair, Flashcard Memory, Sequential Number Memory (Reverse Order), Letter Circle, and Detailed Picture tasks. Eight control participants were not exposed to the TFTA intervention, instead playing games of War, Tic-Tac-Toe, and Hangman, on 4 separate occasions, for 30 minutes, with the investigator. All 16 participants were administered The Dementia Rating Scale-2, The Motor Free Visual Perception Test-4, as well as The Mini Mental State Examination before and after intervention to further assess cognitive skills generalization following the TFTA intervention.

\section{Treatment Effects}

The Five Task Approach utilized a unique combination of therapeutic cueing during sessions 2 through 4 : this cueing highlighted 3 major cognitive skill concepts, The Visual Mental Screen, Chunking, and Cognitive Chaining/Associations. After these cues were introduced to the 8 intervention participants, each of the 8 individuals demonstrated improved scores, increased organization with task approaches, and accelerated task completion times. It is assumed that this drastic increase in participant performance is a direct reflection of exposure to the therapeutic cueing employed. 
The first experimental question addressed the hypothesis that this therapeutic cueing would aide in participants' overall improvement on the 5 TFTA tasks. As previously mentioned, largely concurrent with the investigator's hypothesis, pre and post intervention data for each of the 8 participants revealed largely significant improvements on four of the five tasks. For the one task which did not result in significant improvement, "The Letter Circle Task", it is suspected that significant improvements in participant scores post intervention could not occur presumably because the participants initially performed at a high level on this task, relative to the other tasks, on baseline administration. Thus, performance post-treatment possibly represents a ceiling effect. It should be noted that although a statistically significant difference was not found for this task post intervention, each of the 8 participants improved on the task, all earning a score of $100 \%$ on administration of this game during the last session.

Analysis of the other cognitive 4 tasks, confirmed that participant's improvement post interventional therapeutic cueing was highly significant. Improvements on "The Number Pairs Task" revealed that participants increased by as much as $40 \%$ after cueing was implemented. "The Flashcard Memory Drill” revealed that post cueing, participants scores increased as much as 50\%; with a $40 \%$ increase demonstrated on the "Sequential Number Memory (Reverse Order) Task" post therapeutic cueing. Analysis of "The Detailed Picture Task" revealed as high as a 50\% increase in scores after cues were introduced.

These results are indicative that the clinical cues were indeed beneficial to participant performance, which is reflective of post scores on the 5 tasks. These findings are postulated to be a result of the idea that organizing stimuli in to smaller, and more 
meaningful components, may not only help improve one's memory on tasks such as the TFTA, but further generalize to his or her activities of daily functioning. The therapeutic cue of "the visual mental screen" was implemented in order to encourage the active participants to create a secondary, and personal, memory. Utilizing a visual mental screen when receiving information, as well as when recalling information, has demonstrated increased accuracy and permanency of the memory. Similarly, the other two cueing concepts revolve around combining, or chaining, the stimuli so that seemingly meaningless information becomes somewhat personal to the individual, connected to other facets of the memory, and therefore more difficult to forget.

The process of volitionally utilizing one's visual mental screen is a very taxing task relative to utilization of taxing cognitive processes. Use of visual mental imagery incorporates processes of visual processing and visual perception to the task, as both rely on multiple similar cortical and subcortical regions simultaneously (Ganis, Thompson, \& Kosslyn, 2004). The significant increases in performance observed on all tasks suggests that this brief intervention had positive impact on several areas of cognitive functioning associated with the tasks including working memory, visual and auditory processing, and visual processing speed.

Results of the current study are in congruence with previous research conducted by Fotuhi, Do, and Jack (2012), which demonstrated a positive correlation between engaging in cognitive brain tasks, and subsequent growth of the hippocampus, the major structure within the human brain that is tied to memory. Furthermore, findings of the current study demonstrate that even in elder years, cognitive skills are able to improve when one actively taxes the cortical areas associated with those respective skills. These 
findings are significant for evidence based research as it is understood that both visual and auditory information, as well as general working memory (Glisky, 2007) are cognitive skills that are known to weaken as an individual reaches the later stages of life (Brown et al., 2012; Karbach, 2014).

\section{Standardized Task Outcomes}

As indicated previously, another purpose of this study was to examine generalization of skills addressed in the experimental treatment to improvement on standardized tests associated with these skills. Participants in both the treatment and control groups underwent pre and post intervention administration of the Dementia Rating Scale-2. The second experimental question pertained to the DRS-2, specifically whether exposure to the TFTA intervention would result in increased global cognitive functioning for participants in the active group. Results yielded findings concurrent with the investigator's hypothesis in that the active group demonstrated statistically significant improvements on post-test administration, whereas control participants did not score significantly higher on post-test administration. Relative to the significant findings, one can conclude that the TFTA intervention was potentially responsible for the improvement of the active group in the realm of improved global cognitive functioning, including improvements in generalized skills associated with executive functioning such as attention, initiation/perseveration, and conceptualization. The fact that cognitive training aimed at stimulating pathways (in the already aged brain) can result in generalized improvement effects to other cognitive skill areas that were not targeted (Gates \& Valenzuela, 2010), suggests that brain plasticity is very much present and receptive to intervention, even in one's elder years. 
The third experimental question addressed performance changes on baseline to post intervention administration of the Motor Free Visual Perception Test-4. Findings revealed that active participants showed significant improvement on post administration of the MVPT-4. That is, active participant's demonstrated increased performance in the realm of spatial relationships, visual closure, visual discrimination, visual memory, figure ground, and visual closure skill sets whereas these performance changes were not observed for participants in the control group. It is believed that therapeutic cueing in addition to exposure to the tasks employed by the TFTA resulted in post data reflecting such significant improvements on this specific battery especially because active participants were exposed to both the intervention and simultaneous therapeutic cueing.

Lastly, results of data obtained from pre and post administration of the Mini Mental Status Examination answered the experimental question of whether exposure to the TFTA intervention would yield significant results relative to improvement in areas of global status. Interestingly, both the experimental and control groups demonstrated statistically significant improvement on post administration of this battery. That is, all 16 participants demonstrated increased improvements in the areas of orientation, immediate recall, attention, delayed verbal recall, naming, repetition, reading, writing, and construction, tested on the MMSE. It is possible that there was improvement observed for the control participants because they were forced by essence of participation to been more keen of time and day as to be present for scheduled meetings. It is further speculated that this parallel improvement may be related to the psychometrics of analyzing obtained data in the form of raw scores versus standardized norms. Despite the 
significant performance increases noted for both the active and control groups on the MMSE, these findings are supportive of the notion that cognitive enhancement is possible by engaging in even brief exposures to brain games and therapeutic cueing geared at better organizing presented stimuli. Furthermore, the fact that both groups showed improvement on the MMSE provides further support for Edwards et al. (2005) and Smith et al. (2009) notion that a decline in cognitive functioning does not need to be a continuous, inevitable, or even an irreversible process.

As previously mentioned, it is understood that brain atrophy is often associated with a decline in cognitive functioning, especially with skills mediated by the prefrontal cortex (Anderson et al., 2012). Provided the results of the current study, the TFTA intervention may potentially yield structural cortical changes, in that brain atrophy may remain stagnant, or even reverse current signs of atrophy. In-vivo images taken during the entirety of a duplicated intervention would provide substantial evidence for such claims. Current research conducted by Fotuhi et al. (2012) concluded that cortical structures are indeed susceptible to growth when stimulated, just as they are susceptible to atrophy when left untaxed.

Furthermore, it is understood that as the human brain ages, the most prevalent degree of atrophy is seen in the frontal and temporal lobes. These changes are often present within association cortexes involved with visual and auditory processing, and speed of processing (Fjell, et al., 2014), all of which are skills taxed during the TFTA intervention games. 


\section{Study Limitations}

The methodology of this study was diligently composed with the utmost care to account for confounding variables and variance; as with all research, there were limitations of the study noted. The fact that the study was limited to 16 individuals leaves room for expansion of the research with additional participants. Additionally, the time of day that the participant was seen for each of the sessions was variant, revolving solely around each participant's availability. Ideally, each participant should be seen at the same time of day for the entirety of the study.

Another potential limitation may have been choice of testing, particularly relative to the MMSE. Specifically, in order to have obtained a more comprehensive evaluation of global cognitive status, the investigator may have opted to utilize the Modified Mini Mental State Exam (3MS) (Teng \& Chui, 1987), as opposed to the Mini Mental Status Examination (MMSE) (Folstein et al., 1975). The latter was chosen due to time constraints surrounding completion of the study.

Specifically, because some of the TFTA tasks address auditory processing abilities, inclusion of a standardized test battery measuring auditory processing abilities, pre and post administration, of the TFTA should be considered in duplicated studies as a means of objectively examining generalization of these cognitive skills.

\section{Implications for Future Research}

As assisted living facilities become a more common place for one to permanently retire (Williams, 2011), these facilities should be made aware of the potential benefits of 
incorporating and encouraging cognitive activities such as the TFTA in daily activities. As previously mentioned, a meta-analysis conducted by Sitzer et al. (2006) of selfreported cognitive decline in residents of assisted living facilities was reported to be lower, and reported less frequently, by residents in those facilities where cognitive exercises and activities were included as part of daily living experiences. Future research should also consider skill maintenance relative to the current study. This can be addressed by including a social and functional validation measure as well as periodic monitoring of performance on both the experimental tasks and the standardized measures used to examine generalization.

While the data obtained from the current study is preliminary for evidence based interventions with this population, future research should focus on expanding the intervention to more than 5 cognitive games/tasks. These additional tasks should tax cognitive functions tied to cortical areas shown, and known, to be susceptible to growth after exposure to such tasks. Future research in the form of fMRI of individuals completing such tasks would further our knowledge and understanding of the cortical processes involved with carrying out cognitive activities, and therefore potentially harness these benefits to rehabilitate decreased functioning in individuals. Future research such as the aforementioned is highly warranted at this time in order to provide evidence based research for potential future therapeutic interventions.

\section{Summary and Conclusions}

The results revealed that participants in the active therapeutic condition showed significant improvement in performance on four of the five experimental tasks in the 
TFTA The only task that did not yield significant performance improvement was The Letter Circle Task, and that was due to a potential ceiling effect, as each participant scored very high on baseline administration of the task. On all three standardized batteries, the active group demonstrated statistically significant improvements on post administration. The control group did not show significant improvements on the DRS-2 or on the MVPT-4; however, the control group showed significant improvement on posttesting administration of the MMSE. It is hypothesized that this significant finding was the result of psychometric details in terms of utilizing raw scores for data analysis as opposed to standardized or scaled scores.

The current study provides evidence that even brief exposure to cognitive based therapeutic interventions and cueing may in fact allow for increased performance in a number of cognitive functions. As previously stated, De Gobbi Porto et al. (2015) found that fMRI images after a mere 2 minutes of exposure to cognitive intervention, revealed notable neuroplastic changes in participants, which included changes in the efficacy of synaptic connections, when compared with pre-exposure images. These results in conjunction with the current findings, support the notion that protocols such as the TFTA intervention provide opportunities to engage in activities that stimulate cortical areas which are susceptible to, and potentially demonstrate growth. This is reflective in the active group's post-intervention performance. This type of evidence based research is necessary in order to understand the resurgence of neuroplastic changes, and consequently develop interventions that maximize stimulation of those areas prone to the most potential for increased functioning. 


\section{REFERENCES}

Anderson, K., \& Grossberg, G. (2014). Brain games to slow cognitive decline in alzheimer's disease. The Journal of Post-Acute and Long-Term Care Medicine, 15(8), 536-537.

Anderson, N., Murphy, K., \& Troyer, A. (2012). Living with mild cognitive impairment (pp. 1-10). New York, New York: Oxford University Press.

Bissig, D., \& Lustig, C. (2007). Who Benefits From Memory Training? Psychological Science, 18(8), 720-726.

Brain Games: Test your memory, attention, language skills. (2015, January 1). Retrieved April 1, 2015, from http://www.aarp.org/health/brain-health/brain_games/

Brown, L. A., Brockmole, J. R., Gow, A. J., \& Deary, I. J. (2012). Processing speed and visuospatial executive function predict visual working memory ability in older adults. Experimental Aging Research, 38(1), 1-19 19p.

doi:10.1080/0361073X.2012.636722

Butler, R., \& Lewis, M. (1986). Aging and mental health: Positive psychosocial and biomedical approaches (3rd ed.). Columbus, Ohio: Charles E. Merrill Publishing.

Colarusso, R., \& Hammill, D. (2015). Motor-Free Visual Perception Test-4 (MVPT-4) (4th ed.). Novata, CA: Academic Therapy Publications.

Deary, I. J., Whalley, L. J., Lemmon, H., Crawford, J. R., \& Starr, J. M. (2000). The stability of individual differences in mental ability from childhood to old age: Follow-up of the 1932 Scottish Mental Survey. Intelligence, 28(1), 49-55.

De Gobbi Porto, F. H., Fox, A. M., Tusch, E. S., Sorond, F., Mohammed, A. H., \& Daffner, K. R. (2015). In vivo evidence for neuroplasticity in older adults. Brain Research Bulletin, 114, 56-61. http://doi.org/10.1016/j.brainresbull.2015.03.004

Edwards, J. D., Wadley, V. G., Vance, D. E., Wood, K., Roenker, D. L., \& Ball, K. K. (2005). The impact of speed of processing training on cognitive and everyday performance. Aging \& Mental Health, 9(3), 262-271. doi:http://dx.doi.org/10.1080/13607860412331336788

Eramudugolla, R., Cherbuin, N., Easteal, S., Jorm, A. F., \& Anstey, K. J. (2013). Selfreported cognitive decline on the informant questionnaire on cognitive decline in the elderly is associated with dementia, instrumental activities of daily living and depression but not longitudinal cognitive change. Dementia and Geriatric Cognitive Disorders, 34(5-6), 282-91. doi:http://dx.doi.org/10.1159/000345439 
Fjell, A. M., McEvoy, L., Holland, D., Dale, A. M., \& Walhovd, K. B. (2014). What is normal in normal aging? Effects of aging, amyloid and Alzheimer's disease on the cerebral cortex and the hippocampus. Progress in neurobiology, 117, 20-40. doi:10.1016/j.pneurobio.2014.02.004

Folstein M.F., Folstein S.E., McHugh P.R. (1975). "Mini-mental state: A practical method for grading the cognitive state of patients for the clinician." J Psychiatr Res, 12, 189-198.

Fotuhi, M., Do, D., \& Jack, C. (2012). Modifiable factors that alter the size of the hippocampus with ageing. Nature Reviews Neurology Nat Rev Neurol, 8(4), 189202.

Gaitán, A., Garolera, M., Cerulla, N., Chico, G., Rodriguez-Querol, M., \& Canela-Soler, J. (2013). Efficacy of an adjunctive computer-based cognitive training program in amnestic mild cognitive impairment and Alzheimer's disease: A single-blind, randomized clinical trial. International Journal of Geriatric Psychiatry, 28(1), 9199.

Ganis, G., Thompson, W. L., \& Kosslyn, S. M. (2004). Brain areas underlying visual mental imagery and visual perception: an fMRI study. Cognitive Brain Research, 20(2), 226-241. doi:10.1016/j.cogbrainres.2004.02.012

Gates, N., \& Valenzuela, M. (2010). Cognitive exercise and its role in cognitive function in older adults. Current Psychiatry Reports, 12(1), 20-27.

Glisky, E. (2007). Brain aging: Models, methods, and mechanisms. I. D. R. Riddle (Ed.), Changes in Cognitive Function in Human Aging (pp. 3-20). Boca Raton, Florida: CRC Press.

Harvey, P. D. (2012). Clinical applications of neuropsychological assessment. Dialogues in Clinical Neuroscience, 14(1), 91-99.

Jurica P, Leitten C, Mattis S. (2001). Dementia Rating Scale-2: Professional manual. Lutz: Psychological Assessment Resources.

Karbach, J. (2014). Game-based cognitive training for the aging brain. Frontiers in Psychology, 5, 1100-1101. doi:10.3389/fpsyg.2014.01100

Kirchhoff, B., Anderson, B., Smith, S., Barch, D., \& Jacoby L. (2012). Cognitive training related changes in hippocampal activity associated with recollection in older adults. NeuroImage, 62(3), 1956-1964.

doi:http://dx.doi.org/10.1016/j.neuroimage.2012.06.017 
Leff, B., Kao, H., \& Ritchie, C. (2015). How the principles of geriatric care can be used to improve care for medicare patients. Generations, 39(2) 99-105.

Mulrow, C. D., \& Lichtenstein, M. J. (1991). Screening for hearing impairment in the elderly. Journal of General Internal Medicine, 6(3), 249-258. doi:10.1007/bf02598971

Nouchi, R., Taki, Y., Takeuchi, H., Hashizume, H., Akitsuki, Y., Shigemune, Y., ... Kawashima, R. (2012). Brain training game improves executive functions and processing speed in the elderly: A randomized controlled trial. PLoS ONE, 7(1), e29676. doi:10.1371/journal.pone.0029676

Osaka, M., Yaoi, K., Otsuka, Y., Katsuhara, M., \& Osaka, N. (2012). Practice on conflict tasks promotes executive function of working memory in the elderly. Behavioural Brain Research, 233(1), 90-98. doi:http://dx.doi.org/10.1016/j.bbr.2012.04.044

Rebok, G., Ball, K., Guey, L., Jones, R. N., Kim, H.-Y., King, J. W., ... Willis, S. L. (2014). Ten-year effects of the ACTIVE cognitive training trial on cognition and everyday functioning in older adults. Journal of the American Geriatrics Society, 62(1), 16-24. doi:10.1111/jgs.12607

Roizen, M. F., \& Oz, M. C. (2007). You: Staying young, the owner's manual for extending your warranty Free Press, New York, NY.

Smith, G. E., Housen, P., Yaffe, K., Ruff, R., Kennison, R. F., Mahncke, H. W. \& Zelinski, E. M. (2009), A cognitive training program based on principles of brain plasticity: Results from the improvement in memory with plasticity-based adaptive cognitive training (IMPACT) study. Journal of the American Geriatrics Society, 57: 594-603. doi: 10.1111/j.1532-5415.2008.02167.x

Teng, E., \& Chui, H. (1987). Modified Mini-Mental State Examination. The Journal of Clinical Psychiatry, 48(8), 314-318.

Van Muijden, J., Band, G., \& Hommel, B. (2012). Online games training aging brains: limited transfer to cognitive control functions. Frontiers in Human Neuroscience, 6, 221. doi:10.3389/fnhum.2012.00221

Williams, K. N. (2011), Targeting memory improvement in assisted living: A pilot study. Rehabilitation Nursing, 36: 225-232. doi:10.1002/j.2048-7940.2011.tb00087.x

Zelinski, E. M., \& Gilewski, M. J. (2004). A 10-item Rasch modeled memory selfefficacy scale. Aging \& Mental Health, 8(4), 293- 306. doi:http://dx.doi.org/10.1080/13607860410001709665 


\section{APPENDICES}


Appendix A: Florida International University IRB Approval 


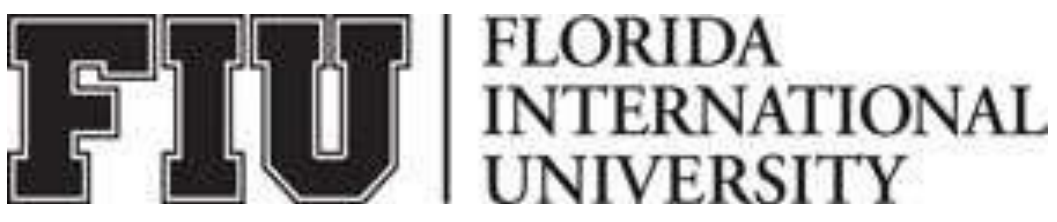

\section{MEMORANDUM}

To:

CC: From:

Date: Protocol Title:

Dr. Monica Hough

File Maria Melendez-Vargas, MIBA, IRB Coordinator

June 10, 2016

"Memory Processing Speed and the Effects of Cognitive Exercise on the Aging Brain"

Office of Research Integrity Research Compliance, MARC 414

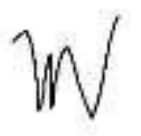

The Health Sciences Institutional Review Board of Florida International University has approved your study for the use of human subjects via the Expedited Review process. Your study was found to be in compliance with this institution's Federal Wide Assurance (00000060).

IRB Protocol Approval \#: IRB-16-0220 IRB Approval Date: 05/09/16 TOPAZ Reference \#: 104740 IRB Expiration Date: $05 / 09 / 17$ 
Appendix B: Visual Screener/Picture Recall Task 


\title{
"Vision Screener-Picture Recall Task"
}

NAME:

DATE:

* Participant must earn $100 \%$ to continue participating in the study.

Purpose: To ensure that the participant is able to read size 20 -font comfortably.

\section{Protocol:}

* Place the paper in front of the participant and have them read each word aloud with no cueing.

\section{Please read the following 5 words aloud.}

\author{
1. APPLE
}

2. GREEN

3. BEACH

4. FLOWER

5. MONKEY 
NAME:

DATE:

* Participant must earn $100 \%$ each session to continue participating in the study.

Purpose: The investigator will assess one's visual perception of color, and visual acuity to ensure that the participant is able to comfortably label colored, 2" by 2" inch images.

\section{Protocol:}

* The particiapnt will verbally label each item, and describe it in brief detail (colors), with no cueing.

Appropriate responses may include:

- Bunny/Rabbit/Hare

- Balloons

- Blue Circle/Blue dot

- Brief case/Suit case/Luggage/Bag

- Loaf of bread/Bread, Muffin 

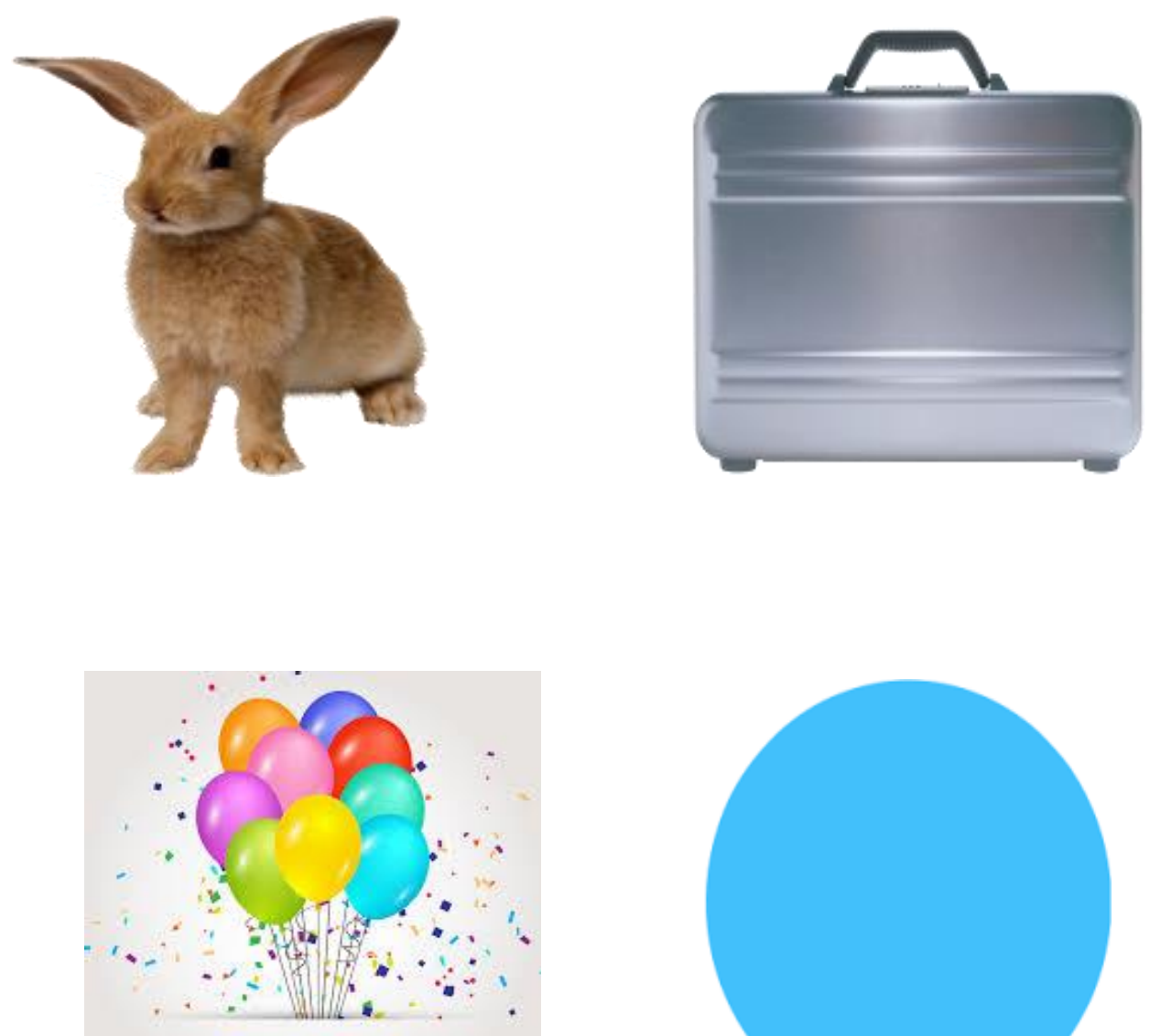

HAPPY BIRTHDAY
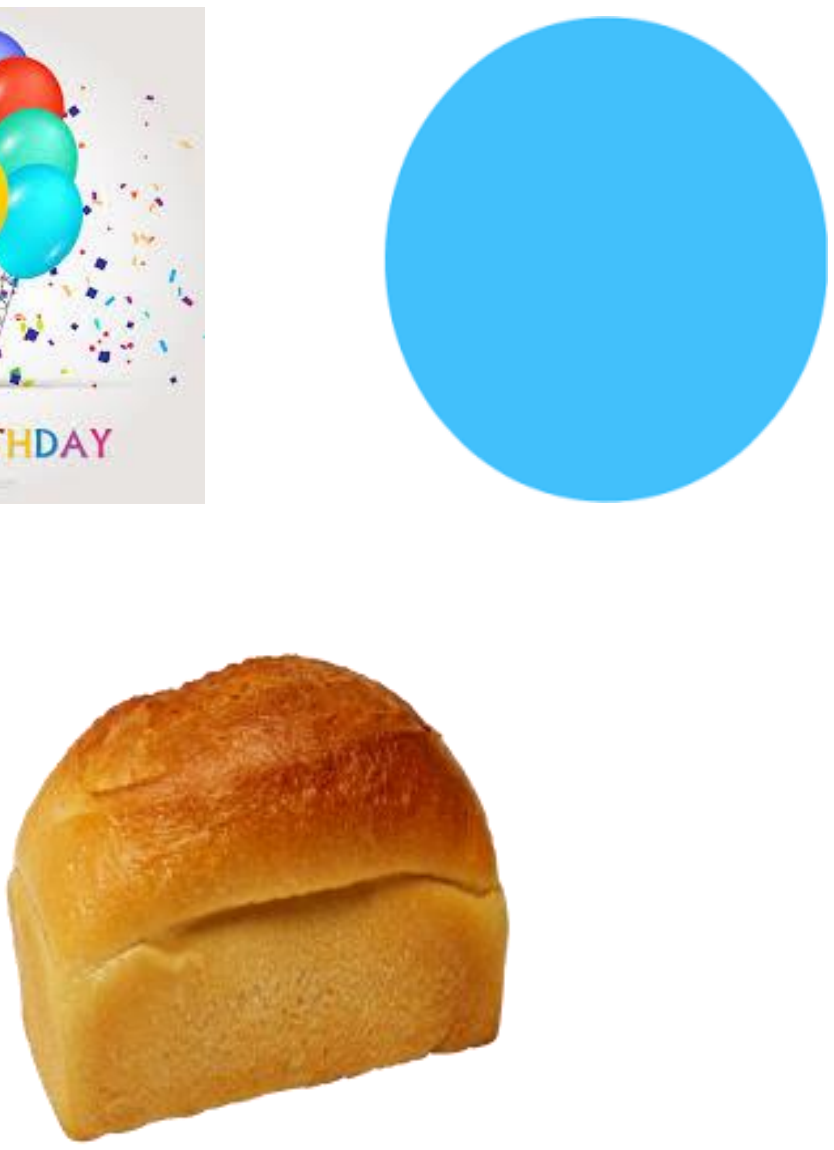
Appendix C: Informed Consent Form 


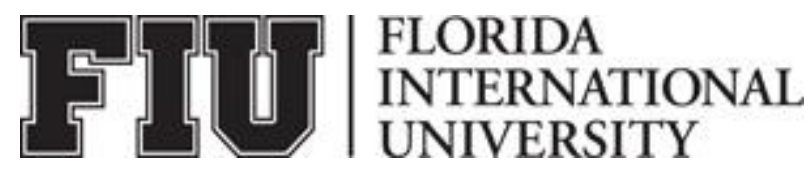

ADULT CONSENT TO PARTICIPATE IN A RESEARCH STUDY

Memory, Processing Speed, and the Effects of Cognitive Exercise on the Aging Brain

PURPOSE OF THE STUDY

You are being asked to be in a research study. The purpose of this study is to see if brain exercises that involve listening and watching will be helpful in improving cognitive skills with a group of older adults. The intervention is called The Five Task Approach (TFTA).

\section{NUMBER OF STUDY PARTICIPANTS}

If you decide to be in this study, you will be one of 16 people in this research study.

\section{DURATION OF THE STUDY}

Your participation will require a maximum of 7 sessions over the course of 2-3 weeks. We will need to meet 2-3 times per week, for 2-3 consecutive weeks. The first and last time that we will meet for approximately 1 hour, the other 5 sessions will last approximately 30 minutes.

\section{PROCEDURES}

If you agree to be in the study, we will ask you to do the following things:

1. Participate in 5 short games, while following verbal instructions.

2. You will be asked to look at and remember different words, letters, numbers, pictures, and listen to sets of numbers.

3. You will be asked to answer questions in reference to the items presented.

- Each session will be audio recorded in order for the data to be analyzed for reliability purposes. One additional investigator will review the recordings.

- You will be informed when the recordings begin, and when the recordings have ended.

\section{RISKS AND/OR DISCOMFORTS}

There are no risks associated with participation in this study.

\section{BENEFITS}

The following benefits may be associated with your participation in this study:

Participants may receive the benefit of increased functioning of cognitive skills, such as listening and following directions, thereby improving quality of life.

\section{CONFIDENTIALITY}


The records of this study will be kept private and will be protected to the fullest extent provided by law. In any sort of report we might publish, we will not include any information that will make it possible to identify a subject. Research records will be stored securely and only the researcher team and Dr. Monica Hough will have access to the records. However, your records may be reviewed for audit purposes by authorized University or other agents who will be bound by the same provisions of confidentiality.

\section{COMPENSATION \& COSTS}

You will not receive any form of compensation for your participation in this study. You may terminate your participation in the study at any time, with no consequence.

You will not be responsible for any costs to participate in this study.

\section{RIGHT TO DECLINE OR WITHDRAW}

Your participation in this study is voluntary. You are free to participate in the study or withdraw your consent at any time during the study. Your withdrawal or lack of participation will not affect any benefits to which you are otherwise entitled. The investigator reserves the right to remove you without your consent at such time that they feel it is in the best interest.

\section{RESEARCHER CONTACT INFORMATION}

If you have any questions about the purpose, procedures, or any other issues relating to this research study you may contact Alexis Yero at Florida International University, (954) 294-6965, Ayero001@FIU.EDU.

\section{IRB CONTACT INFORMATION}

If you would like to talk with someone about your rights of being a subject in this research study or about ethical issues with this research study, you may contact the FIU Office of Research Integrity by phone at 305-348-2494 or by email at ori@fiu.edu.

\section{PARTICIPANT AGREEMENT}

I have read the information in this consent form and agree to participate in this study. I have had a chance to ask any questions I have about this study, and they have been answered for me. I understand that I will be given a copy of this form for my records.

Signature of Participant

Date

Printed Name of Participant

Signature of Person Obtaining Consent

Date 
Appendix D: Pre-session Questions 


\section{"Pre-Session Questions"}

Protocol: The investigator will ask, and record all answers provided by the participant, before the beginning of each session.

1. Yes or no: Have you stopped or started any medications since we last met?

2. Have you sustained any injuries since we last met?

3. Is there anything that has changed in your daily routine since we last met?

4. How are your eyes feeling today, are you seeing colors normally?

5. How are you feeling today on a scale from 1 to 5 , with 5 being one of your "best days?" 
Appendix E: Protocol Explanation of Cues 
- Therapeutic cues to be implemented during therapy sessions 2 through 4 utilizing The Five Task Approach will employ $\underline{3 \text { main principles: }}$

1. "A visual mental screen"

2. "Information chunking"

3. "Information chaining/cognitive associations"

\section{1. "Visual Mental Screen"}

The concept of a "visual mental screen" can best be explained as a "mental dry erase board." Individuals may utilize this "mental screen" by visualizing/projecting both visual and auditory information on to this "screen." It will appear to be a foreign concept to many participants; therefore, the investigator must explain that this mental screen is completely controlled by them. The investigator will tell the participants to imagine the screen as if it is "their mind's eye.". They may ask the participants to verbalize the color, shape, and size that he or she has chosen for their unique mental screen. The investigator may have the participant practice visualizing items on his or her screen before intervention begins. For example, they may have the participants practice varying the color of the screen on command as a means of practicing volitional control of the images they choose to project on to it. Further, the investigator may ask the participant to visualize images such as common objects (apple, car, dog) on his or her mental screen, and have them assign details to the images. Utilization of the visual mental screen will assist participants by providing them with a means of visualizing information in his or her mind, and thereby providing them with a secondary source of a memory or presented stimuli. 


\section{2. "Information Chunking"}

The concept of information "chunking" can best be explained as the grouping of information, while ideally simultaneously visualizing the stimuli on one's visual mental screen. One may choose to "chunk" information in groups of 2 or more items depending on the content of the presented information. In essence, chunking is a means of organizing presented stimuli in to smaller, and more manageable groups of information.

Again, this process is unique to every individual. The investigator will explain that this process is one that is different and most effective for each individual in a different way. The investigator will attempt to assist the participant in a comprehensive understanding of this concept. Consider the following example: If a participant hears the numbers " $2,1,4,3$ ", the investigator will instruct the participant to visualize the numbers 2 and 1 on one side of their screen, and the numbers 4 and 3 on the other side. Other individuals may prefer to chunk the middle 2 numbers and have their screen read 2 independently on the left, with 1 and 4 chunked in the middle, with 3 independently on the right of the screen. Chunking will assist the participant by providing them with a means of visualizing, and organizing, information in his or her mind. Another example may be provided in terms of organizing visual stimuli; that is, if the participant is looking at an image of 7 people standing on a pier, the investigator may encourage the participant to group the individuals into groups of 3 with one odd man out. Perhaps that participant may utilize chunking more effectively by making a group of 4 and a group of 3 individuals in his or her mind. The participant may even opt to mentally underline the middle individual, and chunk the other 6 in to 2 groups of 3 . The investigator will make sure that the participant is clear on this concept before initiation of the second treatment 
session. The use of information chunking will assist participants by providing participants with a means of organizing the information that is presented to them, as opposed to becoming overwhelmed and ignoring details that they could have retained.

\section{3. "Association/Cognitive Chaining"}

The use of information chaining and association can best be explained as the process by which individuals turn presented information in to terms that are unique, personal, and meaningful to them. For example, when an individual is presented with auditory information, they internalize the information by coming up with a story line that connects the words or information to be remembered. An example of this is an individual trying to remember 3 unrelated words: “dog, truck, grass.” He or she may come up with a silly or unique picture that they come up with and visualize on his or her mental screen. The individual could remember those 3 words by visualizing a big $d o g$ driving a small truck on a lawn of freshly cut grass. The story will develop as the words are presented. If there were more items to be remembered, they could continue on with this cognitive image, and develop additional ideas to facilitate memory of the stimulus items. It is thought that the sillier the "story" or image, the harder it will be to forget, and thus the easier it will be to recall.

Another example would be if an individual is trying to remember a string of numbers, he or she may utilize their mental screen, in addition to chunking this information, in order to make the numbers unique and meaningful to the, as a means of aiding with future recall. Perhaps the numbers presented are representative of a meaningful date to that participant, or perhaps if chunked, the numbers are indicative of 
his or her apartment number. These types of associations should be pointed out to the participant. The investigator will make sure that the participant is clear on this concept before initiation of the second treatment session. It is assumed that these associations will facilitate one's recall by providing them with a secondary, individualized memory to rely on when attempting to recall information. The use of cognitive associations will assist participants by providing participants with a means of organizing the information that is presented to them in a unique way that will ideally aid in creating a secondary memory.

Additional clinical cues will require the investigator to provide the participants with positive, verbal encouragement. The investigator will also encourage participants to keep trying even if they are not performing well, and to remain focused. In addition, investigators will encourage participants to work as quickly as possible during the activities, by providing them with intermittent positive, verbal encouragements. For appropriate tasks, the clinician will cue the participant to utilize auditory rehearsal (reiterating information silently to one's self), as well as a horizontal visual search style of the presented stimuli. If the participant appears to approach activities with a disorganized style, and is overtly not implementing the cues, the investigator will remind the participant of cues. Investigators will provide participants with a timing cue, when approximately $50 \%$ of the delineated time is left. That is, on task 1 and 2, investigators will alert the participant when they have 1-minute remaining. On task 3 , participants will be alerted when they have 15 seconds remaining, followed by an alert for task 4 at 30 seconds, and finally, participants will be altered on task 5 when there is 5 seconds remaining. The investigator will utilize all cues at least $1 \mathrm{x}$ each. These clinical cues are assumed to show improvement on post testing measures of the tasks, as well as improve 
the approach a participant will implement when attempting to complete one of the five tasks.

\section{Additional Clinical Cues:}

1. Encouragement to keep trying

2. Reminders to remain focused

3. Encouragement to work as quickly as possible

4. Verbal positive reinforcements

5. Reminders to utilize horizontal image searches

6. Timing cue

7. Auditory rehearsal 
Appendix F: Protocol Participant Cue Script 


\section{"Participant Cue Script"}

The baseline administration of The Five Task Approach (TFTA) will be administered without any cueing from the investigator. The second session of the TFTA will begin with the following explanation of therapeutic cues to be utilized read aloud to the participants. Participants must be encouraged to listen closely, and remember that the goal will be to utilize these 3 cues on subsequent approaches with each of the five tasks.

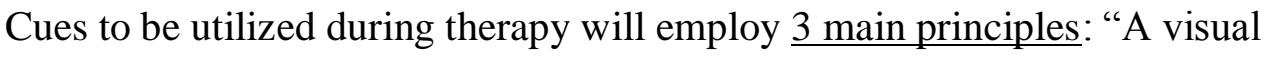
mental screen", "Information chunking", and "Information chaining/cognitive associations.

Protocol: $S L O W L Y$ read the following script to each participant prior to beginning sessions \# 2-5:

"Your Visual Mental Screen"

The concept of a visual mental screen can best be explained as a "mental dry erase board" that is in your mind, and that you control. You may use your mental screen by visualizing, and seeing both visual (gesture to writing) and auditory information (gesture to mouth) that is presented to you on this screen (gesture to center of forehead). This is probably a very silly and foreign concept to you. I am going to explain more; but, for starters, I want you to know that this mental screen is completely controlled by you, and that the more that you practice visualizing things on the screen, the easier it will become. It will assist you by giving you an additional way of remembering information. For example, if I say the word "apple", you may picture a shiny, huge, symmetrical, red apple on your mental screen. Give each item detail and color so that it will be easier to remember. You may also visualize a rotting, lumpy, green, smelly apple. The screen and 
associations you make with the information is entirely controlled by you. The more you practice seeing what you are experiencing on your mental screen, the easier it will become.

EXPLAIN IN DEPTH, AND PROVIDE EXAMPLES AS NEEDED.

\section{"The Concept of Information Chunking"}

The concept of information chunking can be best explained as the grouping of information, while visualizing it on your "visual mental screen." You can chunk information in to groups of 2 items or more. For example, if you heard " $2,1,4,3$ ”, you would visualize the numbers 2 and 1 on one side of your screen, and the numbers 4 and 3 on the other side. If you were presented with 5 numbers, you could underline the $3^{\text {rd }}$ number, as you visualize it on your mental screen, while chunking the other 4 numbers

on each side of the screen (provide a gesture). This will assist you by providing you with a means of visualizing, and organizing, information in your mind.

EXPLAIN IN DEPTH, AND PROVIDE EXAMPLES AS NEEDED.

\section{"Word Associations and Cognitive Chaining"}

The use of information chaining and association can be best explained as the process by which you turn presented information into terms that are unique, and meaningful to you. You make connections and create meaning in otherwise useless information so that you may remember it. For example, when you are presented with auditory information (gesture to ear), you may internalize the information by coming up with a story line that connects the words or the information that you are asked to remember. An example of this is an individual trying to remember 3 unrelated words: 
“dog, truck, grass." Using this tool, he or she may come up with a silly and unique picture or image that they then visualize on their mental screen. They could remember those 3 words by visualizing a huge $d o g$ driving a small truck on a lawn of freshly cut grass. They would add to the story, and create more pictures, as more information was presented to them. The idea is that they created a link, an association, between each item of information, as they made that information unique to them (repeat sentence $1 \mathrm{x}$ ). If there were more items to be remembered, they could continue on with this mental image, and develop additional detailed pictures in order to facilitate memory of the items.

Another example: If I said the words "grass", "mop", and "banana", you could come up with a silly image that is unique to you while you connect the unrelated words with a picture on your mental screen. Perhaps a silly imagine of a fresh cut lawn of green grass has a dirty mop stuck in it with a ripe banana trying to pry it out of the turf. It may seem silly, but try to be as detailed as possible when creating these images on your mental screen, as you try to come up with "connections" that link each word with the next. It is assumed that these mental associations will facilitate your remembering information by providing you with a secondary and unique memory to rely on when attempting to remember the information.

\section{EXPLAIN IN DEPTH, AND PROVIDE EXAMPLES AS NEEDED.}

In addition to the tips we just discussed, I would also like you to be aware that I will be intermittently encouraging you while you play the five games. I will give you verbal encouragements such as "good work", "try to work faster", "keep trying, don’t give up", while you are working on each of the five tasks. I will intermittently remind 
you to stay focused to the task in front of you, while encouraging you to work as quickly as possible. I will encourage you to approach visual tasks in an organized manner. For example, if I present you a series of numbers to analyze, I would like to see you visually search the items in a horizontal fashion. That is, I would like you to look from left to right while you evaluate each item in order. For all five games, I will let you know when you have $50 \%$ of your time remaining. This will help facilitate your concept and passing of time, as well as promote time management, while working on these five tasks. Lastly, I will encourage and remind you to mentally and silently rehearse the items, and words, that you will hear and see, while you also visualize the information on your mental screen. For example, if I said the word "apple", I would like you to visualize a detailed apple that is unique to you, while repeating the word "apple" silently to yourself. You can do this with all of the information I present to you. I know it may seem like a lot to remember, but try to keep all of these tips and tricks in mind while working on each task. I will remind you of all of the cues before each of our sessions together.

Please let me know if you have any questions at this time. 
Appendix G: Number Pairs Activity 


\section{Number Pairs Activity:}

Read the following to the participant: "I am going to present you with 10 rows of numbers. Each row, contains 10 numbers. In each row, there will be 2, and only 2 , of the same exact number. That is, there will be 2 numbers that repeat and match. I want you to work as quickly as possible to find these 2 numbers, and then circle the 2 numbers that you find in each row. After you complete 1 row, quickly move on to the next, until you have complete all of the rows. You may not use your finger or the pen to help you guide your eyes (provide a visual example). Only use your eyes while studying each row. You will have 1 minute to complete this activity. I will tell you when you have 30 seconds remaining. Search for the number pairs in a horizontal fashion, and try not to allow your eyes to become overwhelmed. Take one row at a time. Be sure to silently repeat the number to yourself as your search each row. Do you have any questions? Let's begin."

\section{Instructions:}

* The numbers will be printed in size 30, Times New Roman font. The rows will measure 5' by 2.5 '.

* The investigator will present the participant with 10 rows of numbers, each containing 10 double-digit numbers. Each row has a pair of 2 exact numbers.

* The participant is to find, and circle, the pair of numbers that are exactly the same in each row, as quickly as possible.

* The participant will have 1 minute to complete this task, utilizing the cues specified below.

* If time runs out, all unanswered rows will receive a score of 0 .

* Each correct answer is worth 1 point. Each incorrect, incomplete, or unanswered item will receive a score of 0 .

\section{Protocols:}

* Participants will not be permitted to use his or her finger or pen to track the numbers; instead, they will be instructed to use only his or her eyes to track the numbers. This will promote the use of cortical areas associated with tracking, and visual processing.

* The investigator will record percentage correct for each task.

* The investigator will utilize all cues at least $1 \mathrm{x}$ each.

* Individual performance on each activity will not be disclosed until the end of the study.

Materials: 5 sets of number sheets each consisting of 10 rows of 10 numbers, the answer key, a stopwatch, a pen, 5 scoring forms, and 5 record forms.

Cues: The investigator will cue the participants by: 
- Telling the participant when they have 30 seconds remaining.

- Intermittently providing positive encouragement to the participant to work as quickly as possible. For example, the investigator may say, "You're almost there!" or "You got it!" while the participant is searching for the number pairs.

- Encouraging the participant to rehearse the number in his or her head as each number is being visually scanned.

- Encouraging them to utilize the visual mental screen and to visualize each number on the cognitive screen as they study and scan each digit.

- Encouraging the participant to associate the numbers in terms that are unique to them.

- Encouraging the use of all allotted time, if selection errors are observed. 
Appendix H: Flashcard Memory Drill 
* To print index cards: Set font to size 30, Times New Roman, Bold, Centered, 3' Spacing. Number the cards 1-10, according to session (1-5) on the back on index stapled card.

\section{Flashcard Memory Drill:}

Read the following to the participant: "I am going to present you with 10 flashcards. Each flashcard will contain words of unrelated, concrete objects. I want you to do your best to remember all of the words that you see. While I present you with each card, I will also read the word aloud, and place the card in front of you on the table. I will pause for 2 seconds between each card. I cannot answer any questions related to the game after I present the first card. After I present you all of the $\mathbf{1 0}$ flashcards, I will then remove them from the table, and ask you to recall as many of the $\mathbf{1 0}$ words as you can, within 2 minutes. I will tell you when you have 1-minute remaining. Try to remember the words in order, and to create mental images of each word, even if they are silly or illogical. This will help you associate the random words with a unique meaning to you, and help you remember them easier. The sillier the story the better. Do you have any questions? Let's begin."

\section{Instructions:}

* Participant will be presented 10 flash cards with a word of a concrete object centered in the middle of each. The words will be printed in size $30 \mathrm{font}$, bolded, times new roman, and stapled on a 3 by 5', white index card.

* Participant will attempt to recall all 10 words after he or she has been presented with all of the 10 cards.

* Each card will be read aloud, and presented on a table in front of the participant.

* Cards will be presented with a 2 second delay between each card.

* The cards will be numbered 1-10 on the back, and will be presented in numeric order, in 2 rows of 5.

* Total administration of the 10 cards should take approximately 30 seconds.

* Participants will have 2 minutes to recall the words, utilizing the cues specified below.

* If time runs out, those items not recalled will receive a score of 0.

* Each correct answer is worth 1 point. Each incorrect, incomplete, or unanswered item will receive a score of 0 .

\section{Protocols:}

* The investigator must explain to the participants that they cannot answer questions during the intervention, as that may "give away" the identity of a 
presented card. Do not tell the participant how many words that they have left to recall.

* All repeat responses will be ignored. The participant will not be informed if they have already provided an answer multiple times.

* The investigator will record percentage correct for each task.

* The investigator will utilize all cues at least $1 \mathrm{x}$ each.

* Individual performance on each activity will not be disclosed until the end of the study.

Materials: 50 prepared index cards, a stopwatch, a pen, 5 scoring forms, and 5 record forms.

Cues: The clinician may cue the participants by:

1. Telling the participant when they have 1 -minute remaining.

2. Encouraging the participant to rehearse the word in his or her head as each word is visually scanned. For example, while looking at a word "apple", they would silently rehearse "apple" to themselves.

3. Encouraging the participant to visualize the word on his or her mental screen, while making associations.

4. Instructing the participants to create a chain of unique associations or "silly images", that will also be projected on his or her visual mental screen, and serve to provide them with a secondary memory of each word.

5. Encouraging the use of chunking related words.

6. Encouraging the participant to make meaningful cognitive association relative to each image, while projecting the image on to his or her mental screen.

7. Encouraging the use of all allotted time, if recall difficulty is demonstrated. 
Appendix I: Sequential Number Memory Task (Reverse Order) 
Sequential Number Memory (Reverse Order):

Read the following to the participant: "I am going to read you 4 numbers. I want you to remember those four numbers as best as you can. When I say, "Go", I want you to tell me what those numbers would be in reverse order. For example, if I said “13, 10 (1 - 0), 8, 12", you would say “12, 8, 10 (1 - 0), 13." I will wait 3 seconds before asking you to repeat each digit in reverse order. I will say "go" and point to you when I would like you to begin reciting the number in reverse order. We will do 10 sets of 4 numbers. I cannot repeat certain numbers; I can only repeat the entire set of $\mathbf{4}$ numbers. I can only repeat a set of numbers 1 time, but be sure to ask for a repeat of the numbers if you need. Pay close attention, and repeat the numbers to yourself while writing them on your mental screen. I am not permitted to remind you that I am able to repeat the list of numbers once we begin, so be sure to remember that you are able to ask for one repeat. You will have a maximum of $\mathbf{3 0}$ seconds to repeat the numbers back to me in reverse order. I will tell you when you have 15 seconds remaining. There will be a 2 second pause between each number I read to you. Remember to use the "chunking: technique in which you envision the numbers on your "mental screen", split in to "chunks" of information. Do you have any questions? Let's begin."

\section{Instructions:}

* The investigator will verbally present the participant with 10 sets of 4 numbers (containing both 1 and 2 digit numbers) one at a time. Each set will be presented with a 2 second delay between each response. The participant will wait 2 seconds before reciting the digits in reverse order. Participants will have a maximum of 30 seconds to recall the 4 digits in reverse order.

\section{Protocol:}

* Whenever a number in the number set ends in a zero, the clinician should repeat the number in segments. For example, if the number is 50 , the clinician will say, "50, five, zero" before presenting the next number.

* Clinicians may not repeat only 1 number. If the participant asks for a repeat of any number, the clinician will repeat the set in its entirety $1 x$. That is, each row of numbers may only be repeated $1 x$, if, and only if, requested by the participant. 
* The investigator will remind the participant that they are allowed to hear each number set $2 x$ each, if requested.

* Participants will not be allowed to see the number sheet.

* Individual performance on each activity will not be disclosed until the end of the study.

Materials: 5 sheets of the prepared number sets, a pen, 5 scoring forms, and 5 record forms.

Cues: The clinician may cue the participant by:

1. Telling the participant when they have 15 seconds remaining.

2. To visualize each number on his or her mental screen as they hear each digit.

3. Encouraging the participant to rehearse the number in his or her head as each number is heard. For example, when presented with the number set " $3,5,7,1$ ", they would silently and simultaneously rehearse " $3,5,7,1$ " to themselves.

4. Encouraging "chunking" of the auditory information while utilizing auditory rehearsal, in addition to mentally projecting the numbers on to one's visual mental screen. For example, if the numbers are " $12 / 9 / 8 / 7$, the clinician would instruct the participant to visualize the numbers 12 and 9 on one side of their screen, and the numbers 8 and 7 on the other side, before recalling the numbers in reverse order.

5. Repeating a number set $1 \mathrm{x}$, if requested. 
Appendix J: Letter Circle Task 


\section{Letter Circle Task:}

Read the following to the participant: "I am going to present with you with a sheet of paper filled with upper case alphabetical letters and then tell you a specific letter to search for. It will look similar to a search a word puzzle. I want you to use only your eyes to scan the entire paper carefully, and quickly, while you circle all of the 10 repeated letters. Again, you may not use your finger or the pen to help you guide your eyes. Only use your eyes while studying the paper. Do your best to work as quickly as possible because you will only have 1 minute to search for this letter once I tell you. If you forget the letter I am able to remind you of it 1 time. If you circle the wrong letter by mistake, just cross it out, and quickly move on. I will tell you when you have 30 seconds remaining. Search for the letter in a horizontal fashion, and try not to allow your eyes to become overwhelmed. Say the numbers quickly to yourself as you search each row. Take one row at a time. Do you have any questions? Let's begin.”

\section{Instructions:}

* The clinician should actively watch, and mentally count the correct items circled while the participant is engaged as to record the time it took for them to circle all 10 occurrences.

* Participants will work as quickly as possible to circle all 10 occurrences of a letter the investigator specifies.

* If the participant forgets the letter they are searching for, the investigator may remind them 1 time.

* The participant will have 1 minute to complete this task, utilizing the cues specified below.

* If time runs out, all unanswered rows will receive a score of 0 .

* Each correct answer is worth 1 point. Each incorrect, incomplete, or unanswered item will receive a score of 0 .

\section{Protocol:}

* Participants will not be permitted to use his or her finger or pen to track the numbers; instead, they will be instructed to use only his or her eyes to track the numbers. This will promote the use of cortical areas associated with tracking, and visual processing.

* The investigator will record the time taken to complete the task.

* The investigator will record percentage correct for each task.

* The investigator will utilize all cues at least $1 \mathrm{x}$ each.

* Individual performance on each activity will not be disclosed until the end of the study. 
Materials: 5 prepared letter circle sheets, a pen, a stopwatch, answer key, 5 scoring forms, and 5 record forms.

Cues: The clinician may cue the participant by:

1. Telling the participant when they have 30 seconds remaining.

2. Intermittently providing positive encouragement to the participant to work as quickly as possible. For example, the investigator may say, "You're almost there!" or "You got it!" while the participant is searching for the repeating letters.

3. The investigator may instruct the participant to scan horizontally while searching for the repeating letters. This cue will facilitate lowering visual over load by providing the participant with a method of only dealing with one row at a time.

-This cue would be appropriate if they if the participant appears to be approaching the activity with a disorganized approach.

4. Encouraging the participant to rehearse the numbers in his or her head as each number is being visually scanned. For example, while looking at a row of numbers, the participants will silently repeat the numbers to themselves, as they simultaneously visually process the information.

5. Encouraging them to continue scanning for the entire minute if the participant stops searching before the allotted minute is up without having found all 10 items. 
Appendix K: Detailed Picture Question Task 


\section{Detailed Picture Question Task:}

Read the following to the participant: "I am going to show you 10 different pictures. I want you to study each picture for 10 seconds, one at a time. After the 10 seconds, I am going to remove the picture from sight. I will then ask you a question about that picture. Be sure to pay attention to all of the details in the image. You will have 10 seconds to provide an answer after I ask the question. I cannot answer any questions about the image after I have presented it to you. I will tell you when you have 5 seconds remaining. Remember to visualize the image on your mental screen and make the details as personal as possible. Be sure to use the "chunking" technique as a means of combining the details within each image. Do you have any questions? Let's begin."

\section{Instructions:}

* The investigator will present the participant with an image for 10 consecutive seconds. After the 10 seconds, the image will be removed.

* The participant will provide a response to a detailed question regarding the image presented.

* Images used may be found below. All images are copyright free, and permitted to be moderately reproduced.

\section{Protocol:}

* The investigator must explain to the participants that they cannot answer questions during the intervention, as that may "give away" the identity of any detail within the image.

* The investigator will record percentage correct for each task.

* The investigator will utilize all cues at least $1 \mathrm{x}$ each.

* Individual performance on each activity will not be disclosed until the end of the study.

Materials: 5 prepared sets of 10 pictures (each printed in color on an individual page), a stopwatch, a pen, answer key, 5 scoring forms, and 5 record forms.

Cues: The clinician may cue the participant by:

1. Telling the participant when they have 5 seconds remaining.

2. Encouraging the participant to visualize each image on his or her mental screen as they study it. The investigator may further suggest that the participant attempt to take a "mental picture" of the image and all of its details as they study it.

3. Encouraging the participant to make meaningful cognitive association relative to each image, while projecting the image on to his or her mental screen. 
4. Encouraging "chunking" of the visual information while, in addition to mentally projecting the details on to one's visual mental screen.

5. The clinician may instruct the participant to scan horizontally while studying the images. This cue will facilitate the individual in ensuring that all details of the item are scanned, as they limit visual over load by approaching one area of the image at a time.

6. Providing positive encouragement to the participant toward the end of the 10 seconds if the participant appears to have stopped trying to recall the detail.

7. Encouraging the use of all allotted time to respond to each image, if participant appears to have given up. 
Appendix L: Protocol Control Condition 


\section{Control Condition Protocols}

\section{1. “Hangman" Protocol}

\section{Instructions:}

* Participants will play Hangman with the investigator.

* Only the investigator will provide the phrases for participants to guess.

* If the participant is not familiar, the investigator will explain the concept and teach the strategies to him or her.

* There will be a total of 10 trial guesses for each game. That is, the participant may only guess 10 potential letters before either guessing the phrase, or terminating that round.

* Each round will contain a simple, common phrase. Ex. HAPPY BIRTHDAY.

Materials: Paper, pencils, white board/dry erase board, dry erase pen (optional)

\section{2. "War" Protocol:}

\section{Instructions:}

* The participant and the investigator will play the card game "War" with a deck of jumbo sized font basic playing cards.

* If the participant is not familiar, the investigator will explain the concept and teach the strategies to him or her.

Aces are 1. No wild cards, unless requested.

Materials: 1 deck of basic playing cards with jumbo sized font.

\section{3. "Tic Tac Toe" Protocol:}

\section{Instructions:}

* The participant and the investigator will play Tic Tac Toe.

* If the participant is not familiar, the investigator will explain the concept and teach the strategies to him or her.

* The participant may select whether they would like to be "X" or "O."

Materials: Paper, pencils, white board/dry erase board, dry erase pen (optional) 
Appendix M: Baseline and Record Forms 


\section{TFTA Data Per Session for Tasks \# 1-5 (Appendices G-K)}

NAME:

1G. "NUMBER PAIRS ACTIVITY"

\section{Date:}

\begin{tabular}{|l|l|l|}
\hline $\begin{array}{l}\text { Session 1 } \\
\text { (Baseline) }\end{array}$ & Correct/Incorrect: & $\begin{array}{l}\text { Total Time Taken: } \\
\text { Percentage Correct: }\end{array}$ \\
\hline 1.12 & & \\
\hline 2.77 & & \\
\hline 3.23 & & \\
\hline 4.62 & & \\
\hline 5.33 & & \\
\hline 6.64 & & \\
\hline 7.47 & & \\
\hline 8.96 & & \\
\hline 9.56 & & \\
\hline 10.82 & & \\
\hline
\end{tabular}

Date:

\begin{tabular}{|l|l|l|}
\hline Session 2 & Correct/Incorrect: & $\begin{array}{l}\text { Total Time Taken: } \\
\text { Percentage Correct: }\end{array}$ \\
\hline 1.98 & & \\
\hline 2.12 & & \\
\hline 3.73 & & \\
\hline 4.65 & & \\
\hline 5.13 & & \\
\hline 6.99 & &
\end{tabular}




\begin{tabular}{|l|l|}
\hline 7.17 & \\
\hline 8.62 & \\
\hline 9.50 & \\
\hline 10.21 & \\
\hline
\end{tabular}

Date:

\begin{tabular}{|c|c|c|}
\hline Session 3 & Correct/Incorrect: & $\begin{array}{l}\text { Total Time Taken: } \\
\text { Percentage Correct: }\end{array}$ \\
\hline 1.10 & & \\
\hline 2.41 & & \\
\hline 3.32 & & \\
\hline 4. 19 & & \\
\hline 5.51 & & \\
\hline 6.81 & & \\
\hline 7.11 & & \\
\hline 8.60 & & \\
\hline 9.57 & & \\
\hline 10.35 & & \\
\hline
\end{tabular}

Date:

\begin{tabular}{|l|l|l|}
\hline Session $\mathbf{4}$ & Correct/Incorrect: & $\begin{array}{l}\text { Total Time Taken: } \\
\text { Percentage Correct: }\end{array}$ \\
\hline 1.21 & & \\
\hline 2.27 & \\
\hline 3.79 & & \\
\hline 4.99 & \multicolumn{1}{|l}{} \\
\hline
\end{tabular}




\begin{tabular}{|l|l|}
\hline 5.13 & \\
\hline 6.99 & \\
\hline 7.17 & \\
\hline 8.62 & \\
\hline 9.12 & \\
\hline 10.50 & \\
\hline
\end{tabular}

Date:

\begin{tabular}{|c|c|c|}
\hline $\begin{array}{l}\text { Session } 5 \\
\text { (Post-test) }\end{array}$ & Correct/Incorrect: & $\begin{array}{l}\text { Total Time Taken: } \\
\text { Percentage Correct: }\end{array}$ \\
\hline 1.47 & & \\
\hline 2.78 & & \\
\hline 3.72 & & \\
\hline 4. 60 & & \\
\hline 5.36 & & \\
\hline 6.39 & & \\
\hline 7.82 & & \\
\hline 8.28 & & \\
\hline 9.83 & & \\
\hline 10.10 & & \\
\hline
\end{tabular}


NAME:

2H. "FLASHCARD MEMORY DRILL"

\section{Date:}

\begin{tabular}{|l|l|l|l|}
\hline $\begin{array}{l}\text { Session 1 } \\
\text { (Baseline) }\end{array}$ & $\begin{array}{l}\text { Participant } \\
\text { Response: }\end{array}$ & Correct/Incorrect & Percentage \\
\hline 1. APPLE & Correct: \\
\hline 2. LAMP & & & \\
\hline 3. HAMMER & & \\
\hline 4. DRAPES & & \\
\hline 5.COMPUTER & & \\
\hline 6. BOX & & \\
\hline 7. CANDLE & & & \\
\hline 8. GRASS & & & \\
\hline 9.TELEVISION & & & \\
\hline 10. CUPCAKE & &
\end{tabular}

Date:

\begin{tabular}{|c|c|c|c|}
\hline Session 2 & $\frac{\text { Participant }}{\text { Response: }}$ & Correct/Incorrect & $\begin{array}{l}\text { Percentage } \\
\text { Correct: }\end{array}$ \\
\hline 1. TURKEY & & & \\
\hline 2.JEWELRY & & & \\
\hline 3.CLOTHES & & & \\
\hline 4. PLATE & & & \\
\hline 5. DRESS & & & \\
\hline 6. FLOWER & & & \\
\hline 7. BIKE & & & \\
\hline
\end{tabular}




\begin{tabular}{|l|l|l|}
\hline 8.PEANUT & & \\
\hline 9.CARPET & & \\
\hline 10.PERFUME & & \\
\hline
\end{tabular}

Date:

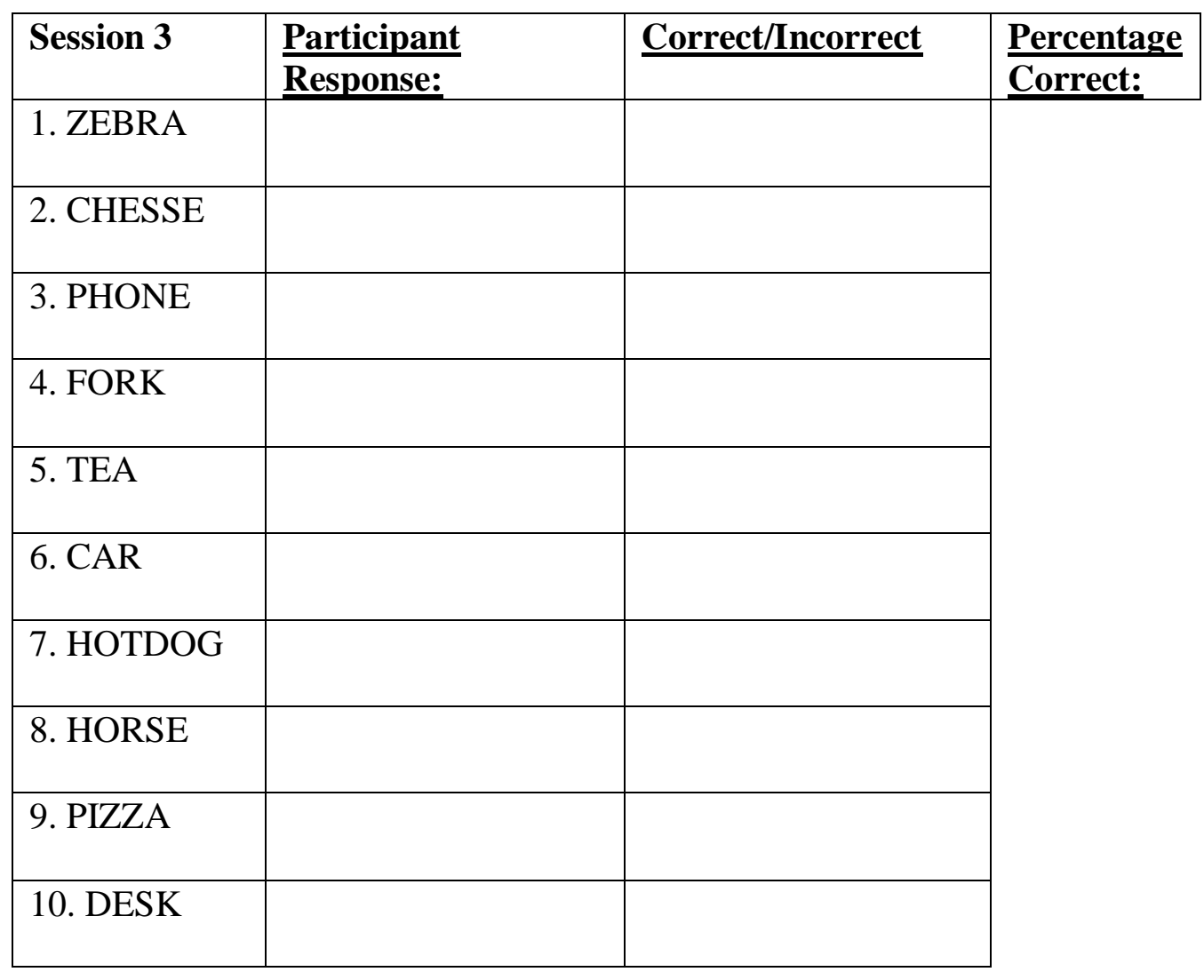

Date:

\begin{tabular}{|c|c|c|c|}
\hline Session 4 & $\begin{array}{l}\text { Participant } \\
\text { Response: }\end{array}$ & Correct/Incorrect & $\begin{array}{l}\text { Percentage } \\
\text { Correct: }\end{array}$ \\
\hline 1. TREE & & & \\
\hline 2. TULIP & & & \\
\hline 3. CANDY & & & \\
\hline 4. FRUIT & & & \\
\hline 5. DOG & & & \\
\hline
\end{tabular}




\begin{tabular}{|l|l|l|}
\hline 6. SOFA & & \\
\hline 7. SPOON & & \\
\hline 8. CHAIR & & \\
\hline 9.RAINBOW & & \\
\hline 10. KEY & & \\
\hline
\end{tabular}

\section{Date:}

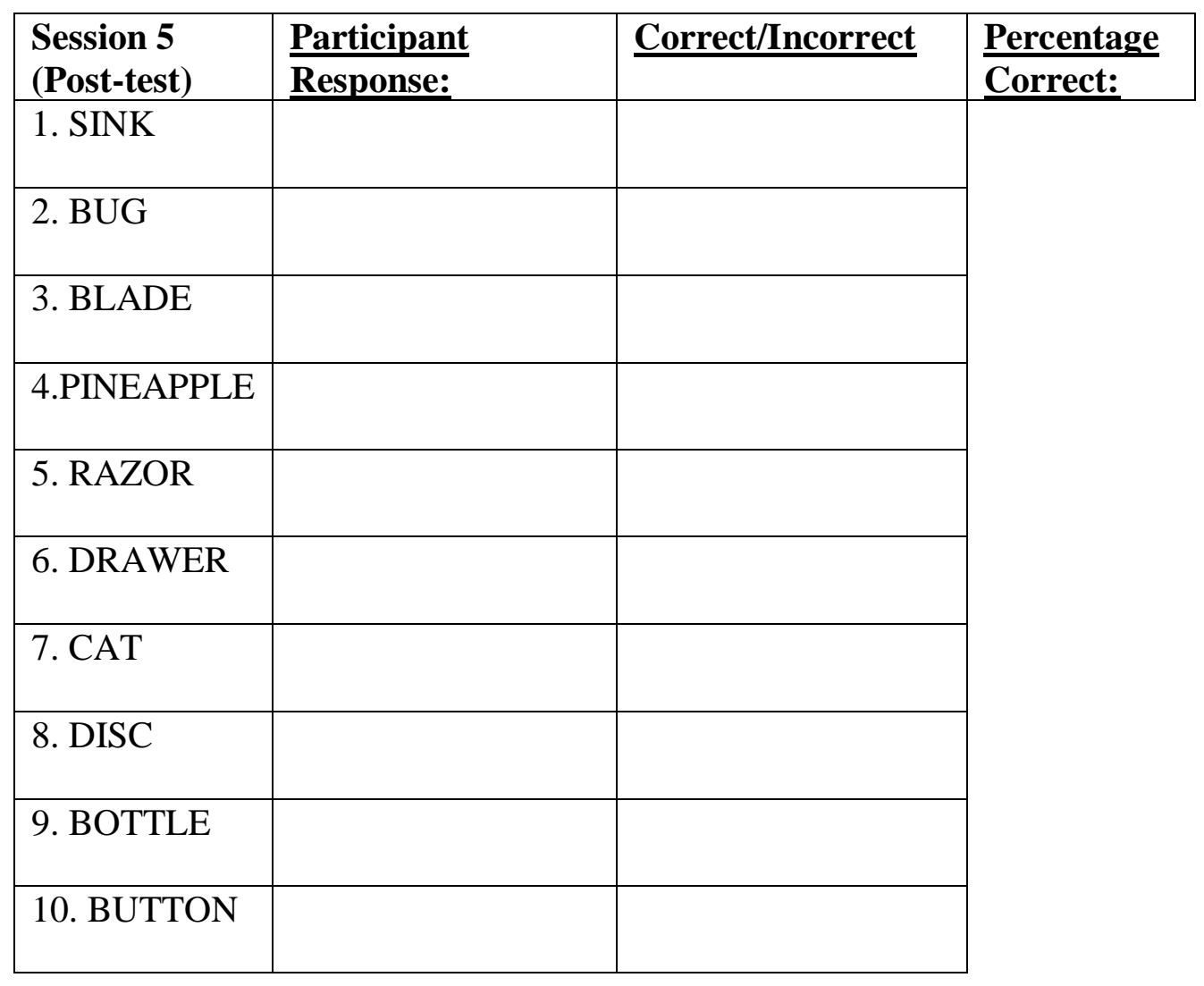


NAME:

3I. "SEQUENTIAL NUMBER MEMORY (REVERSE ORDER)"

Date:

\begin{tabular}{|c|c|c|c|}
\hline $\begin{array}{l}\text { Session } 1 \\
\text { (Baseline) }\end{array}$ & Participant Response: & Correct/Incorrect: & $\begin{array}{l}\text { Percentage } \\
\text { Correct: }\end{array}$ \\
\hline 1. & & & \\
\hline 2. & & & \\
\hline 3. & & & \\
\hline 4. & & & \\
\hline 5. & & & \\
\hline 6. & & & \\
\hline 7. & & & \\
\hline 8. & & & \\
\hline 9. & & & \\
\hline 10. & & & \\
\hline
\end{tabular}

Date:

\begin{tabular}{|c|c|c|c|}
\hline Session 2 & Participant Response: & Correct/Incorrect: & $\begin{array}{l}\text { Percentage } \\
\text { Correct: }\end{array}$ \\
\hline 1. & & & \\
\hline 2. & & & \\
\hline 3. & & & \\
\hline 4. & & & \\
\hline 5. & & & \\
\hline 6. & & & \\
\hline 7. & & & \\
\hline
\end{tabular}




\begin{tabular}{|l|l|l|}
\hline 8. & & \\
\hline 9. & & \\
\hline 10. & & \\
\hline
\end{tabular}

Date:

\begin{tabular}{|c|c|c|c|}
\hline Session 3 & Participant Response: & Correct/Incorrect: & $\frac{\text { Percentage }}{\text { Correct: }}$ \\
\hline 1. & & & \\
\hline 2. & & & \\
\hline 3. & & & \\
\hline 4. & & & \\
\hline 5. & & & \\
\hline 6. & & & \\
\hline 7. & & & \\
\hline 8. & & & \\
\hline 9. & & & \\
\hline 10. & & & \\
\hline
\end{tabular}

Date:

\begin{tabular}{|l|l|l|l|}
\hline Session 4 & Participant Response: & Correct/Incorrect: & $\begin{array}{l}\text { Percentage } \\
\text { Correct: }\end{array}$ \\
\hline 1. & & & \\
\hline 2. & & & \\
\hline 3. & & & \\
\cline { 1 - 2 } & & & \\
\cline { 1 - 2 } & & & \\
\end{tabular}




\begin{tabular}{|l|l|l|}
\hline 5. & & \\
\hline 6. & & \\
\hline 7. & & \\
\hline 8. & & \\
\hline 9. & & \\
\hline 10. & & \\
\hline
\end{tabular}

\section{Date:}

\begin{tabular}{|c|c|c|c|}
\hline $\begin{array}{l}\text { Session } 5 \\
\text { (Post-test) }\end{array}$ & Participant Response: & Correct/Incorrect: & $\begin{array}{l}\text { Percentage } \\
\text { Correct: }\end{array}$ \\
\hline 1. & & & \\
\hline 2. & & & \\
\hline 3. & & & \\
\hline 4. & & & \\
\hline 5. & & & \\
\hline 6. & & & \\
\hline 7. & & & \\
\hline 8. & & & \\
\hline 9. & & & \\
\hline 10. & & & \\
\hline
\end{tabular}


NAME:

4J. "LETTER CIRCLE TASK"

\begin{tabular}{|c|c|c|}
\hline Session 1-5 & Correct out of 10: & \\
\hline $\begin{array}{l}1 . / \mathrm{K} / \\
\text { (Baseline) }\end{array}$ & $/ 10$ & $\begin{array}{l}\text { Date: } \\
\text { Total Time Taken: } \\
\text { Percentage Correct: }\end{array}$ \\
\hline $2 . / \mathrm{F} /$ & $/ 10$ & $\begin{array}{l}\text { Date: } \\
\text { Total Time Taken: } \\
\text { Percentage Correct: }\end{array}$ \\
\hline $3 . / \mathrm{A} /$ & $/ 10$ & $\begin{array}{l}\text { Date: } \\
\text { Total Time Taken: } \\
\text { Percentage Correct: }\end{array}$ \\
\hline 4. /C/ & $/ 10$ & $\begin{array}{l}\text { Date: } \\
\text { Total Time Taken: } \\
\text { Percentage Correct: }\end{array}$ \\
\hline $\begin{array}{l}\text { 5. /H/ } \\
\text { (Post-test) }\end{array}$ & $/ 10$ & $\begin{array}{l}\text { Date: } \\
\text { Total Time Taken: } \\
\text { Percentage Correct: }\end{array}$ \\
\hline
\end{tabular}


NAME:

5K. "DETAILED PICTURE QUESTION TASK"

\section{Date:}

\begin{tabular}{|l|l|l|l|}
\hline $\begin{array}{l}\text { Session1 } \\
\text { (Baseline) }\end{array}$ & Participant Response: & Correct/Incorrect: & $\begin{array}{l}\text { Percentag } \\
\text { e Correct: }\end{array}$ \\
\hline 1. & & & \\
\hline 2. & & & \\
\hline 3. & & & \\
\hline 4. & & & \\
\hline 5. & & & \\
\hline 6. & & & \\
\hline 7. & & & \\
\hline 8. & & & \\
\hline 9. & & & \\
\hline
\end{tabular}

Date:

\begin{tabular}{|c|c|c|c|}
\hline Session 2 & Participant Response: & Correct/Incorrect: & $\begin{array}{l}\text { Percentage } \\
\text { Correct: }\end{array}$ \\
\hline 1. & & & \\
\hline 2. & & & \\
\hline 3. & & & \\
\hline 4. & & & \\
\hline 5. & & & \\
\hline 6. & & & \\
\hline 7. & & & \\
\hline
\end{tabular}




\begin{tabular}{|l|l|l|}
\hline 8. & & \\
\hline 9. & & \\
\hline 10. & & \\
\hline
\end{tabular}

Date:

\begin{tabular}{|c|c|c|c|}
\hline Session 3 & Participant Response: & Correct/Incorrect: & $\begin{array}{l}\text { Percentage } \\
\text { Correct: }\end{array}$ \\
\hline 1. & & & \\
\hline 2. & & & \\
\hline 3. & & & \\
\hline 4. & & & \\
\hline 5. & & & \\
\hline 6. & & & \\
\hline 7. & & & \\
\hline 8. & & & \\
\hline 9. & & & \\
\hline 10. & & & \\
\hline
\end{tabular}

Date:

\begin{tabular}{|c|c|c|c|}
\hline Session 4 & Participant Response: & Correct/Incorrect: & $\begin{array}{l}\text { Percentage } \\
\text { Correct: }\end{array}$ \\
\hline 1. & & & \\
\hline 2. & & & \\
\hline 3. & & & \\
\hline 4. & & & \\
\hline 5. & & & \\
\hline
\end{tabular}




\begin{tabular}{|l|l|l|}
\hline 6. & & \\
\hline 7. & & \\
\hline 8. & & \\
\hline 9. & & \\
\hline 10. & & \\
\hline
\end{tabular}

Date:

\begin{tabular}{|c|c|c|c|}
\hline $\begin{array}{l}\text { Session } 5 \\
\text { (Post-test) }\end{array}$ & Participant Response: & Correct/Incorrect: & $\begin{array}{l}\text { Percentage } \\
\text { Correct: }\end{array}$ \\
\hline 1. & & & \\
\hline 2. & & & \\
\hline 3. & & & \\
\hline 4. & & & \\
\hline 5. & & & \\
\hline 6. & & & \\
\hline 7. & & & \\
\hline 8. & & & \\
\hline 9. & & & \\
\hline 10. & & & \\
\hline
\end{tabular}


Appendix N: TFTA Intervention Data Chart 


\begin{tabular}{|c|c|c|c|c|c|c|}
\hline \multirow[t]{9}{*}{$\begin{array}{l}\text { NUMBER } \\
\text { PAIRS }\end{array}$} & Name & 1 & 2 & 3 & 4 & 5 \\
\hline & $1 \mathrm{AL}$ & 50 & 60 & 70 & 90 & 90 \\
\hline & $2 \mathrm{CM}$ & 60 & 50 & 100 & 100 & 100 \\
\hline & $3 \mathrm{FG}$ & 70 & 60 & 70 & 90 & 70 \\
\hline & $4 \mathrm{MB}$ & 70 & 60 & 70 & 80 & 90 \\
\hline & $5 \mathrm{GW}$ & 40 & 50 & 60 & 90 & 50 \\
\hline & $6 \mathrm{JF}$ & 80 & 70 & 70 & 80 & 100 \\
\hline & $7 \mathrm{DR}$ & 70 & 70 & 80 & 70 & 100 \\
\hline & $8 \mathrm{ML}$ & 50 & 50 & 80 & 70 & 60 \\
\hline \multirow[t]{9}{*}{$\begin{array}{l}\text { FLASH } \\
\text { CARD } \\
\text { MEMORY }\end{array}$} & Name & 1 & 2 & 3 & 4 & 5 \\
\hline & $1 \mathrm{AL}$ & 60 & 80 & 60 & 40 & 70 \\
\hline & $2 \mathrm{CM}$ & 50 & 80 & 80 & 80 & 80 \\
\hline & $3 \mathrm{FG}$ & 30 & 50 & 60 & 50 & 70 \\
\hline & $4 \mathrm{MB}$ & 40 & 70 & 80 & 70 & 90 \\
\hline & $5 \mathrm{GW}$ & 50 & 70 & 60 & 80 & 70 \\
\hline & $6 \mathrm{JF}$ & 80 & 80 & 70 & 100 & 100 \\
\hline & $7 \mathrm{DR}$ & 80 & 50 & 70 & 70 & 70 \\
\hline & $8 \mathrm{ML}$ & 60 & 70 & 70 & 80 & 90 \\
\hline \multirow[t]{9}{*}{$\begin{array}{l}\text { REVERSE } \\
\text { NUMBER }\end{array}$} & Name & 1 & 2 & 3 & 4 & 5 \\
\hline & $1 \mathrm{AL}$ & 30 & 50 & 70 & 40 & 60 \\
\hline & $2 \mathrm{CM}$ & 20 & 20 & 10 & 50 & 40 \\
\hline & $3 \mathrm{FG}$ & 10 & 20 & 20 & 40 & 30 \\
\hline & $4 \mathrm{MB}$ & 60 & 40 & 50 & 70 & 80 \\
\hline & $5 \mathrm{GW}$ & 30 & 40 & 40 & 30 & 50 \\
\hline & $6 \mathrm{JF}$ & 40 & 60 & 60 & 60 & 80 \\
\hline & $7 \mathrm{DR}$ & 20 & 40 & 30 & 50 & 30 \\
\hline & $8 \mathrm{ML}$ & 10 & 30 & 20 & 50 & 50 \\
\hline
\end{tabular}




\begin{tabular}{|llrrrrr|}
\hline LETTER \\
CIRCLE & Name & $\mathbf{1}$ & $\mathbf{2}$ & $\mathbf{3}$ & $\mathbf{4}$ & $\mathbf{5}$ \\
\cline { 2 - 7 } & $1 \mathrm{AL}$ & 100 & 100 & 100 & 100 & 100 \\
\cline { 2 - 7 } & $2 \mathrm{CM}$ & 100 & 100 & 100 & 100 & 100 \\
\cline { 2 - 7 } & $3 \mathrm{FG}$ & 90 & 90 & 100 & 100 & 100 \\
\cline { 2 - 7 } & $4 \mathrm{MB}$ & 100 & 100 & 100 & 100 & 100 \\
\cline { 2 - 7 } & $5 \mathrm{GW}$ & 80 & 90 & 100 & 100 & 100 \\
\cline { 2 - 7 } & $6 \mathrm{JF}$ & 100 & 100 & 100 & 100 & 100 \\
\cline { 2 - 7 } DETAILED & $7 \mathrm{DR}$ & 100 & 100 & 100 & 100 & 100 \\
\cline { 2 - 7 } PICTURE & $8 \mathrm{ML}$ & 90 & 100 & 100 & 100 & 100 \\
\hline & Name & $\mathbf{1}$ & $\mathbf{2}$ & $\mathbf{3}$ & $\mathbf{4}$ & $\mathbf{5}$ \\
\cline { 2 - 7 } & $1 \mathrm{AL}$ & 50 & 60 & 60 & 80 & 70 \\
\cline { 2 - 7 } & $2 \mathrm{CM}$ & 60 & 50 & 50 & 80 & 100 \\
\cline { 2 - 7 } & $3 \mathrm{FG}$ & 60 & 60 & 50 & 50 & 60 \\
\cline { 2 - 7 } & $4 \mathrm{MB}$ & 70 & 80 & 60 & 70 & 80 \\
\cline { 2 - 7 } & $5 \mathrm{GW}$ & 60 & 50 & 50 & 60 & 70 \\
\cline { 2 - 7 } & $6 \mathrm{JF}$ & 40 & 80 & 60 & 70 & 90 \\
\cline { 2 - 7 } & $7 \mathrm{DR}$ & 50 & 60 & 60 & 80 & 70 \\
\cline { 2 - 6 } & $8 \mathrm{ML}$ & 50 & 50 & 70 & 80 & 70 \\
\hline
\end{tabular}

